\title{
Brain-shift compensation using intraoperative ultrasound and constraint-based biomechanical simulation
}

\author{
Fanny Morin ${ }^{\mathrm{a}, \mathrm{b}}$, Hadrien Courtecuisse ${ }^{\mathrm{b}, \mathrm{c}}$, Ingerid Reinertsen ${ }^{\mathrm{d}, \mathrm{e}}$, Florian Le \\ Lann $^{\mathrm{f}}$, Olivier Palombi ${ }^{\mathrm{f}}$, Yohan Payan ${ }^{\mathrm{a}}$, Matthieu Chabanas ${ }^{\mathrm{a}, *}$ \\ ${ }^{a}$ Univ. Grenoble Alpes, CNRS, Grenoble INP, TIMC-IMAG, F38000 Grenoble France \\ ${ }^{b}$ University of Strasbourg, CNRS, AVR-ICube, F67000 Strasbourg France \\ ${ }^{c}$ MIMESIS, INRIA Nancy, France \\ ${ }^{d}$ SINTEF, Department of Medical Technology, Trondheim, Norway \\ ${ }^{e}$ Norwegian National Advisory Unit for Ultrasound and Image-guided Therapy, St. Olav \\ University Hospital, Trondheim, Norway \\ ${ }^{f}$ Grenoble Alpes University Hospital, Department of Neurosurgery, F38000 Grenoble France
}

\begin{abstract}
Purpose. During brain tumor surgery, planning and guidance are based on preoperative images which do not account for brain-shift. However, this deformation is a major source of error in image-guided neurosurgery and affects the accuracy of the procedure. In this paper, we present a constraint-based biomechanical simulation method to compensate for craniotomy-induced brain-shift that integrates the deformations of the blood vessels and cortical surface, using a single intraoperative ultrasound acquisition.
\end{abstract}

Methods. Prior to surgery, a patient-specific biomechanical model is built from preoperative images, accounting for the vascular tree in the tumor region and brain soft tissues. Intraoperatively, a navigated ultrasound acquisition is performed directly in contact with the organ. Doppler and B-mode images are recorded simultaneously, enabling the extraction of the blood vessels and probe footprint respectively. A constraint-based simulation is then executed to register the pre- and intraoperative vascular trees as well as the cortical surface with

\footnotetext{
${ }^{*}$ Corresponding author

Email address: Matthieu.Chabanas@univ-grenoble-alpes.fr (Matthieu Chabanas)
} 
the probe footprint. Finally, preoperative images are updated to provide the surgeon with images corresponding to the current brain shape for navigation.

Results. The robustness of our method is first assessed using sparse and noisy synthetic data. In addition, quantitative results for five clinical cases are provided, first using landmarks set on blood vessels, then based on anatomical structures delineated in medical images. The average distances between paired vessels landmarks ranged from 3.51 to 7.32 (in $\mathrm{mm}$ ) before compensation. With our method, on average $67 \%$ of the brain-shift is corrected (range $[1.26 ; 2.33]$ ) against $57 \%$ using one of the closest existing works (range $[1.71 ; 2.84]$ ). Finally, our method is proven to be fully compatible with a surgical workflow in terms of execution times and user interactions.

Conclusion. In this paper, a new constraint-based biomechanical simulation method is proposed to compensate for craniotomy-induced brain-shift. While being efficient to correct this deformation, the method is fully integrable in a clinical process.

Keywords: Brain-shift, Intraoperative ultrasound, Constraint-based biomechanical simulation, Elastic registration, Boundary conditions, Lagrangian Multipliers.

\section{Introduction}

During brain tumor surgery, accurate localization of the brain tumor is essential to both ensure its total resection and reduce the morbidity of surrounding healthy tissues. Images of the patient's brain are thus acquired prior to surgery 5 and used by neuronavigation systems to assist the surgeon. However, due to the intraoperative deformation of soft tissues, called "brain-shift", these images no longer correspond to brain morphology of the patient throughout the entire procedure.

The impacting factors on the amount and direction of brain-shift are nu10 merous: patient positioning during surgery, craniotomy size and dura opening, 
loss of Cerebrospinal Fluid (CSF), actions of the surgeon, swelling due to drugs, anesthetics or edema, etc. Even if the brain-shift mainly occurs in the direction of gravity, the magnitude is hard to predict and brain deformations are thus estimated by the surgeon. Magnitude and direction of brain-shift have been the subject of several studies reporting cortical displacements up to $20 \mathrm{~mm}$ and subsurface movements up to $7 \mathrm{~mm}$ (Hill et al., 1998, Roberts et al., 1998 , Nimsky et al., 2000, Nabavi et al. 2001). A recent overview of the causes and measurements has been proposed by Gerard et al. (2017).

Brain-shift compensation methods propose to update preoperative images according to the intraoperative deformations of the organ. All of them rely on the acquisition of data during the procedure, providing information about the current tissue deformations. While Magnetic Resonance Imaging (MRI) is the reference preoperative exam, various intraoperative imaging systems are used in the literature such as MRI (Clatz et al., 2005, Vigneron et al., 2012), laser

25 range scanners (LRS) (Sun et al. 2014, Miga et al. 2015), stereo cameras (Sun et al., 2005a) or ultrasound (US) imaging (Reinertsen et al. 2014, Rivaz \& Collins, 2015). However, the data obtained cannot be directly used for surgical navigation: US images are of poor quality compared to preoperative MRI, and surface data alone are not clinically relevant. The goal of brain-shift compen30 sation methods is then to register images acquired before surgery with these intraoperative data. Full brain deformations can thus be recovered based on sparse intraoperative information.

Even if this topic has been widely studied in the literature, very few methods are usable in a clinical practice. Methods aiming to be used in a surgical process have to satisfy three essential criteria. First, acquisition of intraoperative data must be easily integrated in the standard surgical workflow, with limited additional devices and no significant increase of the operative time and cost. Then, the compensation method must be relevant in terms of execution times and as automatic as possible. This criteria should be valid for every step of 40 the proposed method, including image data pre-processing (e.g. patient-specific biomechanical model preparation) and intraoperative computations. Finally, 
accuracy and robustness must obviously be validated on actual surgical cases.

In this paper, we present a method that intends to fulfill these requirements. First, we chose to rely on the acquisition of US images during surgery. Compared to other intraoperative imaging systems, US is portable, compatible with other surgical equipment and already available in most operating rooms. In addition, US provides subsurface information and not only the cortical surface. While soft tissues can be viewed using B-mode US, the Doppler mode enables specific imaging of the blood vessels. Next, a constraint-based biomechanical simulation method is proposed to compute the MR/US registration. Displacements are imposed over a finite element (FE) brain model to account for the deformations of the vascular tree and the exposed cortical surface.

The contributions of this work are summarized below:

1. We propose to account for the deformation of the blood vessels and exposed cortical surface using a single intraoperative US acquisition (Doppler and B-mode images recorded simultaneously).

2. The whole compensation method is fully detailed, including the data extraction from MR and US images and constraint-based biomechanical simulation. The choices regarding the construction of the brain model, the constraints definition and their filtering are described. Our work combines these specific technical contributions with existing ones within a new complete craniotomy-induced brain-shift compensation system.

3. The performance of our method is evaluated over synthetic and patient data. For five clinical cases, a quantitative analysis is proposed first using landmarks set on blood vessels, then based on anatomical structures (such as sulci or ventricles) delineated in intraoperative B-mode images.

4. Improvements, in terms of accuracy and robustness, compared to one of the closest existing method (Reinertsen et al., 2014) are presented.

5. Finally, our method is fully compatible with a surgical process in terms of user interactions and execution times.

This article is organized as follows. First, detailed related works are pre- 
sented in Section 2. Then, the method is described in the four following sections. Experiments on synthetic and patient data are detailed in Section 7, and the results are presented in Section 8 . Finally, the method and results are discussed 75 in Section 9 .

\section{Related works}

After a description of the existing brain-shift compensation methods, this section focuses on the biomechanical simulation of brain deformations.

\subsection{Brain-shift compensation methods for brain tumor surgery}

The main methods to compensate for brain-shift occurring during tumor surgery can be classified as image-based and biomechanical simulation-based techniques. These approaches are presented in the following subsections. Each of them is organized according to the kind of intraoperative data.

\subsubsection{Image-based registration methods}

Nimsky et al. (2001) presented a rigid registration between pre- and intraoperative MR images, using fiducial markers placed around the craniotomy. For their part, a non-linear registration method based on mutual information was introduced by Hastreiter et al. (2004). Regardless of the accuracy, the use of such intraoperative MR devices is cumbersome. The acquisition procedure is complex and increases the operating time considerably: the patient must be transferred to the scanner and specific surgical tools are required due to the magnetic field generated. Moreover, these devices are expensive and require large dedicated operating rooms. For these reasons, this intraoperative modality is thus rarely used in clinical routine.

${ }_{95}$ Pereira et al. (2016) proposed to use pre- and intraoperative Cone-Beam Computed Tomography (CBCT) enhanced with intravenous injection of contrast agent. The volumetric deformation vector field was computed combining first a rigid registration of the skull then an elastic transformation based on 
vessels, ventricles and image intensities. However, CBCT images suffer from ever, a clinical validation using data from 5 patients (Reinertsen et al. 2007b) showed that no major improvements are provided by the elastic registration technique over the rigid one. Finally, this rigid registration approach was vali- 


\subsubsection{Biomechanical simulation-based registration methods}

Key surfaces, such as the cortical surface and the surface of the lateral ventricles, were tracked in pre- and intraoperative MR exams by Ferrant et al. (2001) and Ferrant et al. (2002). Displacements were ating microscope. Their system was evaluated on 10 patients.

¿ Next, similar methods were presented by Mostayed et al. (2013) and Garlapati et al. (2014). Sparse point sets of the exposed part of the brain were 


\subsection{Brain biomechanical simulations}

A recent survey focusing on brain biomechanical simulation has been proposed by Morin et al. (2017). In this section, works dealing with the determination of the mechanical properties of brain tissues are first introduced, pointing 
havior laws is then nuanced in a second paragraph, according to the kind of loadings applied on the brain during simulations. Finally, the main biomechanical models published in the literature are presented.

\subsubsection{Mechanical properties}

195 sponding parameters) have been widely studied in the literature. These properties can be determined using rheological studies on animal or human brains. Experimental measurements, obtained by compression, tension or suction of the tissues, are correlated with numerical simulation using a biomechanical model.

Even if a non-linear behavior of the organ is highlighted by the studies cited in the next paragraph, the equivalent Young's modulus $E$ at small deformations and the Poisson's ratio $\nu$ are given for comparison.

Among studies realized on porcine brains, Paulsen et al. (1999) introduced a biphasic poroelastic model where the organ was considered as a sponge-like material. It was modeled using a porous solid tissue $(E=2100 \mathrm{~Pa}$ and $\nu=0.45)$ with interstitial fluid. This hypothesis was nuanced by Tavner et al. (2016) who demonstrated that there was no bulk fluid flow through the brain in-vivo. The authors argued that computing such a biphasic model was unnecessary and a single phase viscoelastic model should provide enough accuracy. Based on swine brains observations, Miller \& Chinzei (2002) proposed an Ogden-like hyper-viscoelastic model with a Prony-series relaxation modulus. The very low stiffness of porcine brain tissues was also pointed out by Kaster et al. (2011), reporting Young's modulus for the white and grey matter respectively equal to $1787 \pm 186 \mathrm{~Pa}$ and $1195 \pm 157 \mathrm{~Pa}$. Finally, the only rheological experiment on an in vivo human brain was presented by Schiavone et al. (2009). The authors used a light aspiration device and proposed a modified 2-term Mooney-Rivlin law, with an equivalent $E=1440 \mathrm{~Pa}$ and $\nu=0.45$.

Brain stiffness can also be determined in vivo using MR Elastography (MRE). This imaging technique estimates the shear modulus $\mu$ from harmonic shear 
son's ratio by $E=2 \mu(1+\nu)$, the value of $E$ corresponding to the measured $\mu$ can be computed for indication (here with a $\nu=0.45$ ). With the measurements reported by Kruse et al. (1999); Uffmann et al. (2004); McCracken et al. (2005); Kruse et al. (2008), the estimated Young's moduli were significantly grey matter) than the ones found with rheological experiments. However, the estimated $\mu$ decreased with the shear wave frequency (Chatelin et al., 2012). For numerical simulation, a static Young's modulus (corresponding to a null excitation frequency, to avoid any viscosity effect) is required. Then, the Young's moduli computed above probably overestimate this static value.

\subsubsection{Boundary conditions and loadings}

While getting an accurate description of the tissues is essential, boundary conditions (e.g. contacts with the dura mater) and loadings are at least as important for the accuracy of comprehensive simulations. These loadings can be applied through forces or displacements.

On the one hand, Valencia et al. (2012) applied a pressure (i.e. a force field) on the surface of a brain model, while running the simulation with various elastic and hyper-elastic constitutive laws. Relevant differences are shown for resulting displacements and especially stresses at equilibrium. Moreover, Morin 240 et al. (2015) pointed out the importance of accounting for the internal prestress of the organ. Indeed, the study exhibited significant differences for the resulting displacements when applying the same free force over a pre-stressed and an unpre-stressed brain model. In case of force loading, the biomechanical model is thus sensitive to the constitutive law and parameters, including internal pre-stress.

On the other hand, Wittek et al. (2009) and Miller \& Lu (2013) investigated the impact of tissues mechanical properties when loadings are imposed through displacements. The authors showed that the solution in displacement is weakly sensitive to the chosen constitutive laws and parameters. In that sit- 

mechanical properties.

\subsubsection{Biomechanical models}

Many biomechanical models of the brain have been proposed in the literature in order to compensate for brain-shift, to simulate the growth of a pathological eled as homogeneous, although some authors have integrated local properties. For example, Castellano-Smith et al. (2003) set different stiffness parameters to the white and grey matter, and Miller \& Lu (2013) and Mostayed et al. 
In addition, very soft and compressible properties are assigned to the ventricles ( $E=10 \mathrm{~Pa}$ and $\nu=0.05$, against $E=694 \mathrm{~Pa}$ and and $\nu=0.45$ for brain tissues) by Clatz et al. (2005) and Bucki et al. (2012), in order to simulate the CSF loss during brain-shift deformation. This CSF is thus modeled as a liquid (2011)

For the constraints, Dumpuri et al. (2006) proposed an automatic method to generate the boundary conditions. While the upper part of the brain, close to the craniotomy, is stress free, the lower cerebellum part is fixed. Sliding constraints are applied on the remaining brain surface, similarly to the ones imposed between the brain and the skull by Prastawa et al. (2009). Similar constraints are also used by Chen et al. (2011) to simulate interactions between the brain and the dural septa (falx cerebri and tentorium cerebelli). A very complex model was also introduced by $\mathrm{Hu}$ et al. (2007) where the dura mater isotropic elastic shell elements with high stiffness.

Finally, most of the biomechanical models mentioned in the above paragraphs rely on finite element (FE) meshes. Their generation includes soft tissues segmentation and then meshing steps that can be time-consuming. In order to avoid these limitations, brain biomechanical simulations based on meshless techniques (Miller et al., 2012, Zhang et al. 2013) were proposed in the literature. Even if a slight segmentation step is still needed, no meshing process is thus required.

\subsection{Conclusion}

305

In terms on intraoperative data our method rely on ultrasound images, to us the best compromise between provided data (surface and subsurface information, about tissues and vessels) and practical use in the operating room. For the MR/US registration, we propose a biomechanical simulation-based approach to extrapolate sparse deformation data to the entire brain based on a physical and 


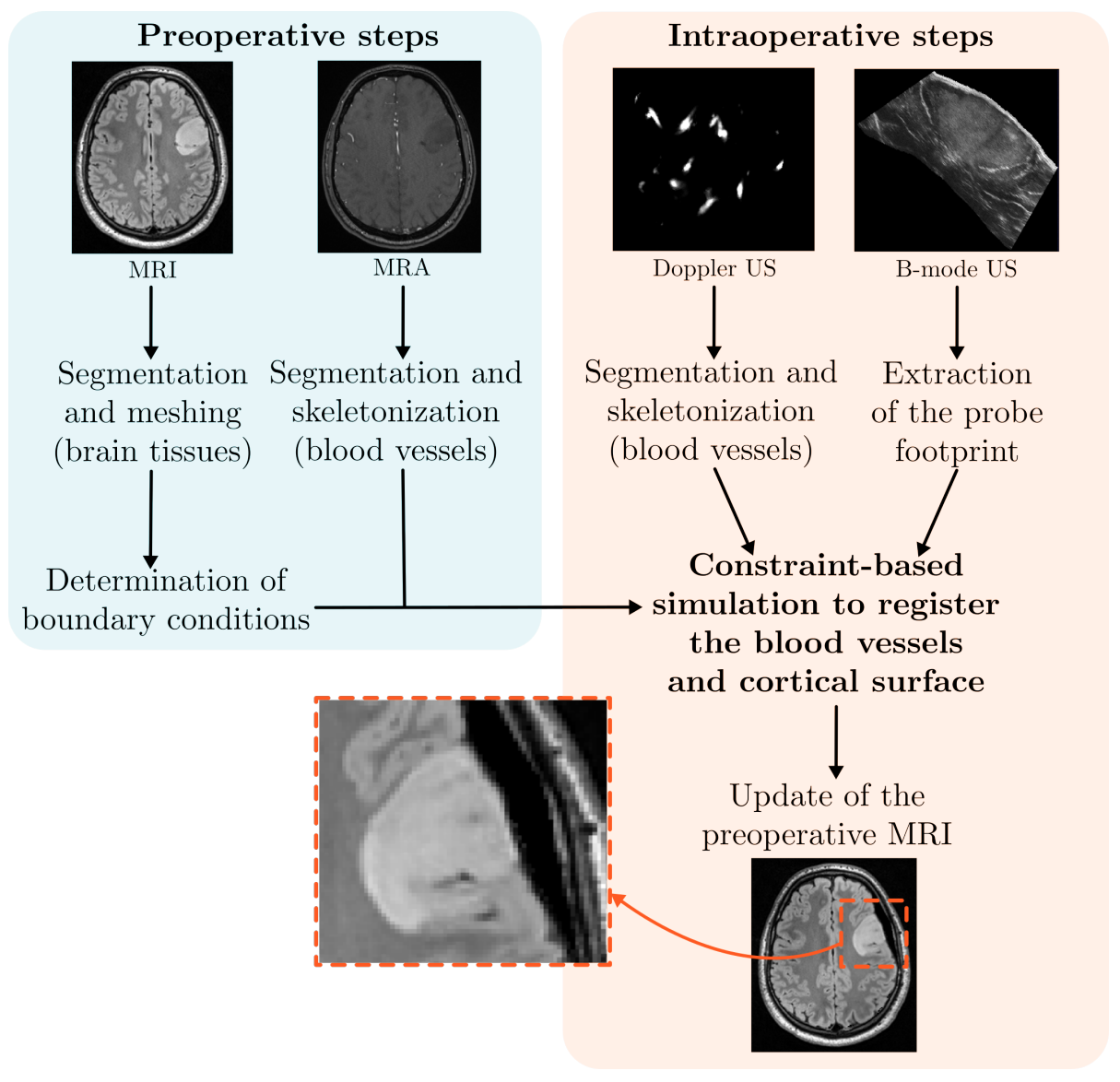

Figure 1: Brain-shift compensation process

not only geometrical background. Our modeling choices are based on the above review of the literature.

\section{Method overview}

First, the overall process of our method is described. Second, the modeling choices made to perform the registration are detailed.

The overall process of our method is presented in Figure 1. Preoperatively, an anatomic patient-specific brain model is built from the images acquired prior 
to surgery. Brain soft tissues and blood vessels around the tumor are extracted from MRI and MRA respectively. Boundary conditions (mainly contacts with the dura mater, falx cerebri and tentorium cerebelli) are then determined and transferred to the generated brain model. As preoperative images are often acquired one day before surgery, several hours are therefore available to build the brain model. However, the generation of the patient-specific model should be as automatic as possible.

Intraoperatively, a series of navigated 2D US slices is acquired directly in contact with the brain after opening the skull and dura mater (Reinertsen et al. 2014). Power Doppler as well as B-mode information are recorded simultaneously. A single US acquisition is thus necessary to obtain the whole intraoperative data. Two 3D volumes are reconstructed from the Doppler and B-mode slices, enabling the extraction of the blood vessels and probe footprint respectively. A constraint-based biomechanical simulation is then used to perform the registration of the pre- and intraoperative vascular trees and of the probe footprint with the surface of the brain model. Finally, the preoperative MRI is updated using the displacement field computed over the brain model. In order to comply with a clinical workflow, the designed extraction algorithms have to be robust, fast and must limit user interactions.

\subsection{Brain modeling}

Figure 2 illustrates the modeling choices and the use of each component during the constraint-based biomechanical simulation.

The brain is represented using several models, each one being optimized for a different task (upper panel in Figure 22). First, a deformable model describes the internal mechanical behavior of the soft tissues. Its geometry is represented by a tetrahedral FE mesh. Next, two collision models, a brain surface mesh and a skeleton of the blood vessels, are exclusively employed to express the Lagrangian Multipliers used to impose displacements (i.e. constraints) on the brain. During FE resolution, constraint forces are then computed so that the imposed displacement are satisfied at the end of the simulation step, whatever 


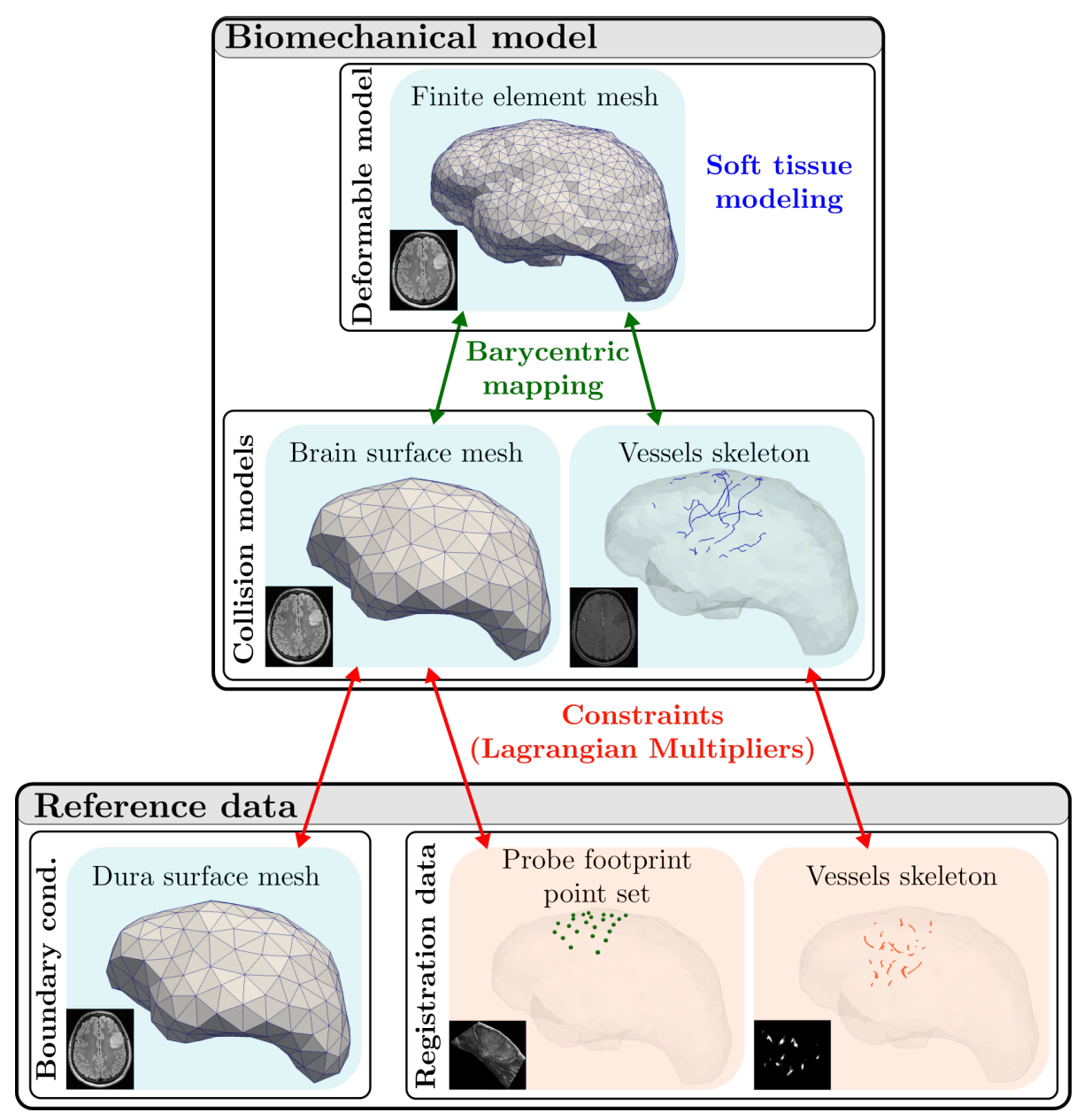

Figure 2: Different components and data types of our brain model. Their interaction and use are highlighted. Preoperative and intraoperative data are respectively shown within a blue and orange box. 
the biomechanical properties of the model. The deformable and collision models are coupled using a barycentric mapping.

The reference data (lower panel in Figure 2) are used as a base to express the constraints forces. These data are not deformed and remain fixed during all the simulation. First, the dura surface mesh is employed to express the boundary conditions between the surface of the organ and the dura mater. Then, the registration data (i.e. the probe footprint point set and the vessels skeleton) are extracted from intraoperative US images. They are used to drive the deformation of the biomechanical model to compensate for brain-shift.

The technical background that is necessary to understand our constraintbased biomechanical simulation is first detailed in Section 4 . The extraction and generation methods of the meshes, skeletons and point sets are then described in Section 5, highlighting the brain modeling choices. A last section presents the constraint-based biomechanical simulation, focusing on the main technical contribution of this paper: the definition of the Lagrangian Multipliers constraints and their filtering.

\section{Simulation background}

After introducing the corotational formulation and barycentric mapping, this section details the static governing equations used for the simulation of the brain and their numerical resolution. While these notions are not original to this paper, they are essential to understand our constraint-based simulation method.

\subsection{Corotational formulation}

First introduced in the field of computer graphics (Müller et al. 2002), this formulation is frequently used for medical simulations due to its low computational cost. For such a formulation, the elastic forces applied on the nodes of a tetrahedral element of the brain FE mesh can be written as:

$$
\mathbf{f}_{e}=\mathbf{R}_{e} \mathbf{K}_{e}^{l}\left(\mathbf{R}_{e}^{T} \overline{\mathbf{u}}_{e}-\mathbf{u}_{e}\right)
$$


where $\mathbf{u}_{e}$ and $\overline{\mathbf{u}}_{e}$ are respectively the initial and deformed positions of the four nodes of the tetrahedron and $\mathbf{f}_{e}$, the elastic forces applied on these nodes. While $\mathbf{K}_{e}^{l}$ corresponds to the local linear stiffness matrix of the element, parametrized by the Young's modulus $E$ and the Poisson's ratio $\nu, \mathbf{R}_{e}$ is a bloc diagonal matrix defined as:

$$
\mathbf{R}_{e}=\left[\begin{array}{cccc}
\mathbf{R} & & & 0 \\
& \mathbf{R} & & \\
& & \mathbf{R} & \\
& & & \mathbf{R}
\end{array}\right]
$$

with $\mathbf{R}$ being the $3 \times 3$ rotation matrix of the tetrahedral element. The rotation of each element, computed based on the $Q R$ decomposition proposed by Nesme et al. (2005), is evaluated independently accounting for finite rotations. Swelling artifacts showed by classic linear elasticity are thus removed.

The local stiffness matrix $\mathbf{K}_{e}$ can be written with the synthetic formulation (Felippa \& Haugen, 2005):

$$
\mathbf{K}_{e}=\mathbf{R}_{e} \int_{V_{e}}\left(\mathbf{C}_{e} \mathbf{D}_{e} \mathbf{C}_{e} \partial V_{e}\right) \mathbf{R}_{e}^{T}
$$

where $\mathbf{C}_{e}$ and $\mathbf{D}_{e}$ are the strain-displacement matrix and the stress-strain matrix respectively. While these matrices are constant during the simulation, $\mathbf{R}_{e}$ needs to be updated at each iteration.

\subsection{Barycentric mapping}

As described above, several meshes and skeleton are used to describe the brain model (see Figure 2). Soft tissue modeling is solved over the deformable model whereas the constraint forces are computed and applied on the vertices of the collision models. During each resolution step, the computed constraint forces (i.e. Lagrangian Multipliers) have thus to be transferred from the collision models to the FE model. Conversely, once the deformation is computed, the FE nodes displacements must be propagated to the collision vertices. As a result, a barycentric mapping is used to link the different representations of the brain. 
As proposed by Faure et al. (2012), each vertex of the collision models is associated with its closest FE tetrahedron at the beginning of the simulation. Since the collision and FE models are deformed at the same time, this association remains constant over the simulation. A vertex position can thus be defined by a linear combination of the nodes positions of its associated tetrahedron using barycentric coefficients. All these linear combinations are gathered by the function $\mathcal{J}$, used to map the positions $\mathbf{u}_{F E}$ of the deformable model to the positions $\mathbf{u}_{c o l}$ of the collision models:

$$
\mathbf{u}_{c o l}=\mathcal{J}\left(\mathbf{u}_{F E}\right)
$$

Using the Jacobian matrix of this function, defined as $\mathbf{J}=\partial \mathbf{u}_{c o l} / \partial \mathbf{u}_{F E}$, equation (4) can be written as:

$$
\mathbf{u}_{c o l}=\mathbf{J} \mathbf{u}_{F E}
$$

This Jacobian matrix is thus used to deduce $\mathbf{u}_{c o l}$ from the positions $\mathbf{u}_{F E}$.

For the constraint forces $\boldsymbol{\lambda}_{c o l}$ applied to the collision models, the mapping computes and accumulates the equivalent forces on the FE nodes. The transferred constraint forces $\boldsymbol{\lambda}_{F E}$ are then weighted with the same barycentric coefficients using:

$$
\boldsymbol{\lambda}_{F E}=\mathbf{J}^{T} \boldsymbol{\lambda}_{c o l}
$$

In the following sections, the notation $\mathbf{u}$ and $\boldsymbol{\lambda}$ will refer to the nodes positions of the FE brain model $\left(\mathbf{u}=\mathbf{u}_{F E}\right)$ and constraint forces (i.e. Lagrangian Multipliers $)$ applied on this model $\left(\boldsymbol{\lambda}=\boldsymbol{\lambda}_{F E}=\mathbf{J}^{T} \boldsymbol{\lambda}_{c o l}\right)$ respectively.

\subsection{Governing equation}

The governing equation is given by the static formulation:

$$
\mathcal{F}(\mathbf{u})+\mathcal{H}(\mathbf{u}) \boldsymbol{\lambda}=\mathbf{f}_{\text {ext }}=0
$$

where $\mathcal{F}(\mathbf{u})$ and $\mathcal{H}(\mathbf{u})$ are both non-linear functions providing the internal forces and gathering the constraint directions for the positions $\mathbf{u}$ respectively. In our case, no external forces $\mathbf{f}_{\text {ext }}$ (e.g. gravity or CSF pressure) are applied to the model. 
Instead of directly solving this non-linear problem, each simulation step $i$ consists in solving a linearized problem according to the two unknown $\mathbf{u}$ and $\boldsymbol{\lambda}$. Note that computing the solution of this linearized problem is equivalent to performing the first iteration of the Newton-Raphson algorithm for the static problem (7). After several simulation steps, the positions at equilibrium (i.e. $\Delta \mathbf{u}^{i}=\mathbf{u}^{i+1}-\mathbf{u}^{i}=0$ ) correspond to the solution positions of this formulation.

The linearized equation is given by:

$\mathcal{F}\left(\mathbf{u}^{i}\right)+\mathcal{H}\left(\mathbf{u}^{i}\right) \boldsymbol{\lambda}^{i}+\left(\left.\frac{\partial \mathcal{F}}{\partial \mathbf{u}}\right|_{\mathbf{u}^{i}}+\left.\frac{\partial \mathcal{H}}{\partial \mathbf{u}}\right|_{\mathbf{u}^{i}} \boldsymbol{\lambda}^{i}\right)\left(\mathbf{u}^{i+1}-\mathbf{u}^{i}\right)+\mathcal{H}\left(\mathbf{u}^{i}\right)\left(\boldsymbol{\lambda}^{i+1}-\boldsymbol{\lambda}^{i}\right)=0$

At the beginning of each iteration step, no constraint forces are applied to the FE model which yields $\boldsymbol{\lambda}^{i}=0$ and then:

$$
\mathcal{F}\left(\mathbf{u}^{i}\right)+\left.\frac{\partial \mathcal{F}}{\partial \mathbf{u}}\right|_{\mathbf{u}^{i}}\left(\mathbf{u}^{i+1}-\mathbf{u}^{i}\right)+\mathcal{H}\left(\mathbf{u}^{i}\right) \boldsymbol{\lambda}^{i+1}=0
$$

On the one hand, the global stiffness matrix of the model is defined as:

$$
\mathbf{K}^{i}=\left.\frac{\partial \mathcal{F}}{\partial \mathbf{u}}\right|_{\mathbf{u}^{i}}=\sum\left(\mathbf{G}_{e} \mathbf{K}_{\mathbf{e}}^{i} \mathbf{G}_{e}^{T}\right)
$$

where $\mathbf{G}_{e}$ is the globalization matrix transferring the local stiffness matrix $\mathbf{K}_{\mathbf{e}}^{i}$ of an element $e$ to the global stiffness matrix $\mathbf{K}^{i}$. Since the rotation matrices of the corotational formulation are not constant (see Section 4.1), $\mathbf{K}^{i}$ must be recomputed at each simulation step.

On the other hand, the formalism of the Lagrangian Multipliers is used to express the constraints. A collision detection is first performed at the beginning of each iteration. $\mathcal{H}\left(\mathbf{u}^{i}\right)$ then remains constant during all the step allowing to define the constraint matrix $\mathbf{H}^{i}$ as:

$$
\mathbf{H}^{i^{T}}=\mathcal{H}\left(\mathbf{u}^{i}\right)
$$

The number of lines in $\mathbf{H}^{i}$ is equal to three times the total number of constraints in the simulation. For each constraint, this matrix gathers the weighted normals. The core of the constraint-based biomechanical simulation proposed in this paper is then to define the contents of the matrix $\mathbf{H}^{i}$ (collision detection and weighed normal computation). 
Once $\mathbf{H}^{i}$ is defined, a constraint law can be written as:

$$
\boldsymbol{\delta}=\tilde{\mathbf{H}}^{i} \mathbf{p}^{i}-\mathbf{H}^{i} \mathbf{u}
$$

with $\boldsymbol{\delta}$ being the violation of constraints, $\mathbf{p}$ the targeted positions of the constraint and $\tilde{\mathbf{H}}^{i}$ the matrix of the unweighted constraint normals. The linearization of this constraint law in $\mathbf{u}^{i}$ gives:

$$
\boldsymbol{\delta}^{i+1}=\tilde{\mathbf{H}}^{i} \mathbf{p}^{i}-\mathbf{H}^{i} \mathbf{u}^{i}-\mathbf{H}^{i}\left(\mathbf{u}^{i+1}-\mathbf{u}^{i}\right)=\mathbf{c}^{i}-\mathbf{H}^{i}\left(\mathbf{u}^{i+1}-\mathbf{u}^{i}\right)
$$

where $\mathbf{c}^{i}=\tilde{\mathbf{H}}^{i} \mathbf{p}^{i}-\mathbf{H}^{i} \mathbf{u}^{i}$ remains constant during each iteration.

The final linearized equation of (7) is obtained by injecting equations 10 ) and (11) in equation (9):

$$
\mathbf{K}^{i} \boldsymbol{\Delta} \mathbf{u}^{i}+\mathbf{H}^{i T} \boldsymbol{\lambda}=-\mathcal{F}\left(\mathbf{u}^{i}\right) \quad \text { with } \quad \boldsymbol{\Delta} \mathbf{u}^{i}=\mathbf{u}^{i+1}-\mathbf{u}^{i}
$$

where the unknowns are $\boldsymbol{\Delta} \mathbf{u}^{i}$ and $\boldsymbol{\lambda}$.

For the sake of simplicity, superscript $i$ is omitted in the following paragraphs, which describe the resolution for a single step only.

\subsection{Numerical resolution}

Rewriting equations (14) and 13 provides the following Karush-KuhnTucker (KKT) system:

$$
\left\{\begin{array}{l}
\mathbf{K x}+\mathbf{H}^{T} \boldsymbol{\lambda}=\mathbf{b} \\
\mathbf{c}-\mathbf{H x}=\boldsymbol{\delta}
\end{array}\right.
$$

where $\mathbf{x}=\boldsymbol{\Delta} \mathbf{u}$ and $\mathbf{b}=-\mathcal{F}(\mathbf{u})$. At each iteration, the KKT problem is solved in five steps with the Schür complement method.

\subsubsection{Free motion}

We first compute the free deformation $\mathbf{x}_{\text {free }}=\mathbf{K}^{-\mathbf{1}} \mathbf{b}$, that does not depend on $\boldsymbol{\lambda}$, using a preconditioned Conjugate Gradient (Courtecuisse et al., 2011). The fixed Dirichlet conditions imposed on the base of the brainstem ensure that $\mathbf{K}$ is invertible. Injecting $\mathbf{x}_{\text {free }}$ in equation 15 gives:

$$
\mathbf{x}=\mathbf{x}_{\text {free }}-\mathbf{K}^{-\mathbf{1}} \mathbf{H}^{T} \boldsymbol{\lambda}
$$




\subsubsection{Collision detection}

A proximity-based detection is performed for each constraint, allowing to define the matrix $\mathbf{H}$ described above. The free violation of constraint $\boldsymbol{\delta}_{\text {free }}$ is defined as $\boldsymbol{\delta}_{\text {free }}=\mathbf{c}-\mathbf{H x}_{\text {free }}$, with $\mathbf{x}_{\text {free }}$ being the node positions of the brain model if no constraint force $\boldsymbol{\lambda}$ is applied.

\subsubsection{Compliance computation}

Injecting (17) in (16) gives:

$$
\boldsymbol{\delta}_{\text {free }}+\mathbf{H K}^{-1} \mathbf{H}^{T} \boldsymbol{\lambda}=\boldsymbol{\delta}
$$

${ }_{425}$ allowing to define the compliance matrix $\mathbf{W}=\mathbf{H K}^{-1} \mathbf{H}^{T}$ which traduces the mechanical coupling of the constraints. Its computation is the most time consuming step in the iteration since it involves the inversion of the large matrix $\mathbf{K}$. In order to get a clinically time relevant simulation, $\mathbf{K}^{-\mathbf{1}}$ is approximated by $\mathbf{K}^{0^{-1}}$, with $\mathbf{K}^{0}$ being the stiffness matrix at the first iteration. $\mathbf{K}^{0^{-1}}$ is computed preoperatively and stored as a dense matrix. As the mechanical coupling (due to small brain stiffness parameters, see Section 2.2.1) and the brain deformations are low, this approximation is consistent in our simulation case.

\subsubsection{Constraint solving}

Equation (18) thus provides the Non Linear Complementarity Problem:

$$
\mathbf{W} \boldsymbol{\lambda}=\boldsymbol{\delta}-\boldsymbol{\delta}_{\text {free }}
$$

where both $\boldsymbol{\lambda}$ and $\boldsymbol{\delta}$ are unknown. The problem is solved using a modified

Gauss-Seidel algorithm as detailed by Duriez et al. (2006).

\subsubsection{Corrective motion}

Once the constraint forces $\boldsymbol{\lambda}$ are known, they are replaced in equation 15 giving:

$$
\mathbf{x}=\mathbf{K}^{-\mathbf{1}}\left(\mathbf{b}-\mathbf{H}^{T} \boldsymbol{\lambda}\right)
$$

As for the Free Motion step, this equation is solved using a preconditioned Conjugate Gradient providing the final positions $\mathbf{u}^{i+1}$ that fulfill the constraints. 


\section{Brain modeling and data extraction from medical images}

This section is divided into two subsections describing how pre- then intraoperative medical images are processed to build the brain models respectively.

\subsection{Anatomic patient-specific brain model generated from preoperative images}

\subsubsection{Soft tissues segmentation}

Soft tissues are segmented from preoperative MRI (in our case a T2-FLAIR sequence). The whole brain is first extracted using the fast and automatic method proposed by Smith (2002). A region growing algorithm is then used to segment the tumor. Finally, the hemispheres are separated and the brainstem, cerebellum and falx cerebri are segmented by an operator.

\subsubsection{Soft tissues meshing}

For the FE brain mesh, only the hemisphere affected by the tumor is accounted for. Soft tissues, excluding the cerebellum, are uniformly meshed with tetrahedrons. The surface of the previous FE mesh is used to represent the brain surface collision mesh. It is then decimated in order to reduce the number of constraints during the simulation and thus consequently reduce computation time. Finally, the same decimated surface mesh is used to describe the dura mesh.

\subsubsection{Determination of boundary conditions}

Boundary conditions are used to simulate the interactions between the brain and its surrounding anatomical structures. On the one hand, sliding contacts between the brain surface collision model and dura mater, including the falx cerebri and tentorium cerebelli, are accounted for and illustrated by Figure 3 . On the other hand, FE nodes located at the base of the brainstem are fixed during the simulation.

For all these boundary conditions, indexes of the constrained nodes are automatically selected. To do so, the corresponding brain model meshes are compared to segmentation volume boundaries of each anatomic element. The mesh vertices close to this surface are then selected. 


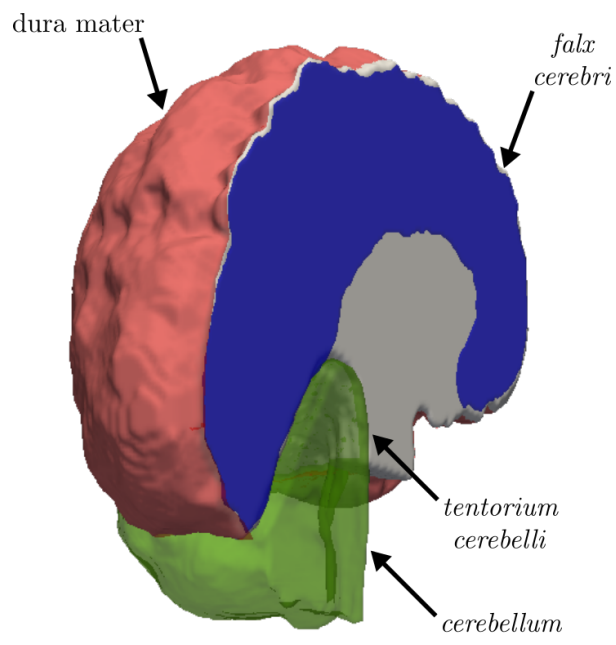

Figure 3: Boundary conditions applied on the brain surface collision mesh. The segmentation volumes of the dura mater, falx cerebri and tentorium cerebelli are respectively shown in red, blue and green. Sliding contacts are applied on the mesh vertices close to these volumes.

\subsubsection{Segmentation of the vascular tree}

Blood vessels are segmented from the preoperative MRA exam using the Maximum of Intensity Projection (MIP) image analysis method adapted from the one proposed by Vermandel et al. (2007). After segmenting targeted vascular structures, the binarized MIP image is used as a mask to recover 3D positions of vessels from MRA 2D slices.

In order to get more contrast in the region of interest, the MRA is cropped to tumor region before computing the MIP image. Two peaks are then visible in the histogram of the MIP image, corresponding to the background (black) and the soft tissues (dark grays) respectively. The blood vessels, highlighted with a contrast product, are thus represented by the higher intensities. The MIP image is therefore segmented with a threshold set just after the second peak of the histogram. The segmented MIP image is shown in Figure 4(a).

However, artificial noise can appear due to the 3D reconstruction of the segmented volume. In order to increase the robustness of our vessels extraction method, the whole segmentation process is performed for the $\mathrm{x}, \mathrm{y}$ and $\mathrm{z}$ di- 


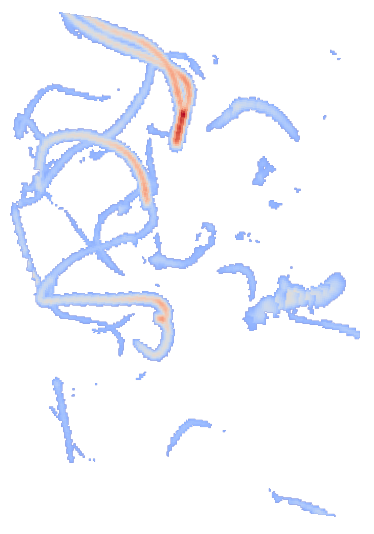

(a) Segmented MIP image

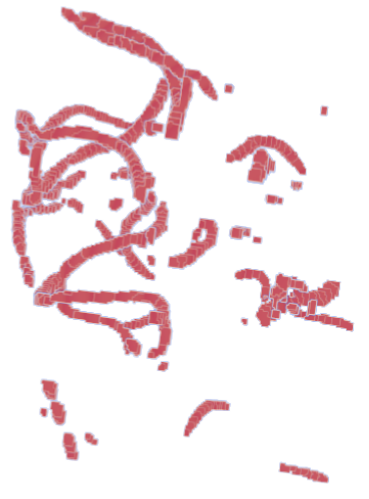

(b) Segmented volume

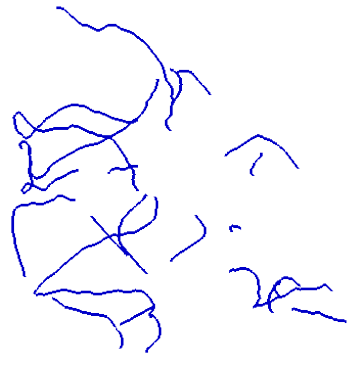

Figure 4: Blood vessels extraction from MRA

rections of the 3D space, thus obtaining three segmentation volumes. Finally, these volumes.

\subsubsection{Skeletonization of the vascular tree}

The blood vessels skeletonization process is based on the modified Dijkstra algorithm detailed by Wan et al. (2002). First, the Euclidean distance to the closest vessel boundary (DBF-distance) is computed for each voxel inside the vessels. Images are then converted into a connected graph, using the inverse value of the DBF-distance. Finally, vascular branches are extracted using a modified Dijkstra algorithm (Figure 4(c)).

As the regularity of the extracted skeleton strongly depends on the quality and voxel size of the input segmented image, each branch is smoothed and re-sampled using B-splines. Finally, points and their connectivity (edges) are exported. 


\subsection{Data extraction from intraoperative US images}

\subsubsection{Blood vessels skeleton extraction}

500 these images provide high contrast information on the blood vessels, their segmentation is simply achieved using a threshold set by an operator (Reinertsen et al. 2007b). Finally, the skeletonization step is realized with the algorithm described in Section 5.1.5. operative vessels skeleton and probe footprint point set (see Figure 2). The number of constraints will then be linked to the number of points composing these elements. While a high number of constraints has a strong impact on the computation times, it can also lead to an over-constrained simulation. They are

\subsubsection{Probe footprint point set extraction}

The probe footprint point set is extracted from the B-mode US images. As the B-mode volume is performed (image acquisition and 3D volume reconstruction) at the same time as the Doppler one, its quality is quite low compared to a dedicated B-mode acquisition. However, as the probe footprint is easily identifiable (localization in the US image, pixels intensities and plane geometry), such image quality is sufficient to automatically extract it.

Since the probe footprint is localized on the edge of the B-mode US volume, the boundaries of this volume are first extracted. In order to only keep the highest intensities, this surface is then segmented using a threshold set by an operator. While the probe footprint is extracted, some noise, spread over the surface of the B-mode volume, also remains. The obtained binarized volume is therefore sampled and turned into a graph where close points are connected. Finally, the largest connected component is extracted.

\subsubsection{Data decimation}

As mentioned above, constraints are directly expressed based on the intratherefore decimated in order to reduce the number of constraints. With respect 
to the resolution of the FE and surface collision meshes, the blood vessels skeleton and the probe footprint point set are sampled at 1 point per $2.5 \mathrm{~mm}$ and 1 point per $10 \mathrm{~mm}$ respectively.

\subsubsection{Deactivation of boundary conditions at the craniotomy location}

Vertices on which boundary conditions are applied are automatically determined preoperatively (see Section 5.1 .3 for details). However, the position and size of the craniotomy are unknown before surgery. When the dura mater is opened, contacts between the brain and this membrane at the craniotomy location have therefore to be deactivated (i.e. corresponding vertices have to be removed from the list of the constrained indexes).

Intraoperatively, the craniotomy region is assimilated to the area where US slices are acquired, and then, to the probe footprint location in US images. The extracted point set is thus rigidly registered with the brain surface using the ICP algorithm (Besl \& McKay, 1992). Finally, all the boundary conditions close to the probe footprint are deactivated.

\section{Constraint-based biomechanical simulation}

The constraint-based biomechanical simulation used to compensate for brainshift is described in this section. First, the choices about the soft tissues modeling are briefly justified. Next, this section focuses on the main technical contribution of our registration method: the definition of the constraints applied on the collision models and their filtering. Finally, the update of the preoperative images with the simulated brain deformations is succinctly described.

\subsection{Soft tissue modeling}

As detailed in Section 2.2.1, brain mechanical behavior is complex and should be described using hyper-elastic laws. In our context, displacements are imposed on the brain model to simulate the contacts with the dura mater, register the blood vessels and constrain the cortical surface. The solution in displacements should thus be weakly sensitive to the biomechanical properties 
(see Section 2.2.2 for details). A linear elastic law is therefore accounted for.

In addition, the corotational formulation has the strong advantage to avoid the swelling artifacts observed with pure linear elastic models while requiring low computational efforts (see Section 4.1 for details). This formulation thus appeared to us as being a good trade-off in our context. For the parametrization, $E$ and $\nu$ are respectively set to $1.5 \mathrm{kPa}$ and 0.45 , following Schiavone et al.

60 2009). A higher stiffness equal to $10 \mathrm{kPa}$, close to the value chosen by Miller $\& \mathrm{Lu}(2013)$, is used for the tumor region.

\subsection{Constraints}

On the one hand, constraints are used to solve the contacts between the brain and dura mater, including the falx cerebri and tentorium cerebelli, and between the brain and probe footprint. On the other hand, displacements are imposed through forces applied on the blood vessels to register of the pre and intraoperative vascular trees. For all these constraints, the same formalism of Lagrangian Multipliers is used (see Section 4.3): constraint forces $\boldsymbol{\lambda}$ are computed in order to cancel the violation of constraints $\boldsymbol{\delta}$. This section thus describes how the matrix $\mathbf{H}$ is filled, especially detailing the collision detection and weighted normals computation phases for each constraint type. Even if some parts of this process could be improved, for example by detecting collisions in a more efficient way (Joldes et al. 2009), this is beyond the scope of this paper.

As mentioned above (see Figure 2), collision detection and response are computed over the collision models. In the next paragraphs, the constraint forces $\boldsymbol{\lambda}$ thus correspond to $\boldsymbol{\lambda}_{c o l}$ defined in Section 4.2. Vertices positions of the collision meshes, before and after the constraint forces are applied, are noted $\mathbf{u}$ and $\overline{\mathbf{u}}$ respectively and drawn in red and green in the figures. They are associated with fixed target positions chosen among the reference data meshes ${ }_{580}$ (see Figure 2) called $\mathbf{p}$ and drawn in blue. Finally, the violation of constraints after the resolution is noted $\overline{\boldsymbol{\delta}}$. 


\subsubsection{Bilateral dura constraint $\mathbf{\Omega}$}

This constraint is used to simulate interactions between the brain and the folds of the dura mater. It is applied on the vertices of the brain surface mesh in contact with the falx cerebri and tentorium cerebelli. The collision detection is processed between these vertices and the dura surface mesh. Each vertex, with position $\mathbf{u}_{j}$, is associated with a target position $\mathbf{p}_{j}$, computed as the closest projection of $\mathbf{u}_{j}$ over the Bezier surface computed over the dura mesh. Since the dura mesh is fixed, Bezier coefficients are evaluated once for each triangle on the beginning of the simulation. This is used to obtain smooth contacts with the membrane, with constraints being continuous all along the surface.

The violation of constraint $\boldsymbol{\delta}_{j}$ is computed as:

$$
\boldsymbol{\delta}_{j}=\mathbf{n}_{j} \cdot\left(\mathbf{p}_{j}-\mathbf{u}_{j}\right)
$$

with - being the Euclidean dot product and $\mathbf{n}_{j}$ the normalized normal to the Bezier surface at position $\mathbf{p}_{j}$. During the constraint solving step (described in Section 4.4.4, the corresponding constraint force $\boldsymbol{\lambda}_{j}$, applied in the direction of $\mathbf{n}_{j}$, is computed so that no violation remains $\left(\overline{\boldsymbol{\delta}}_{j}=0\right)$ in that direction.

After the resolution, the vertex $j$, with position $\overline{\mathbf{u}}_{j}$, is located on the tangential plane given by $\mathbf{n}_{j}$ (see Figure $5(\mathrm{a})$ for details). During the simulation, the brain model then remains in contact with the falx cerebri and the tentorium cerebelli but is authorized to slide along these surfaces.

\subsubsection{Unilateral dura constraint $\Psi$}

This constraint is applied to the brain surface collision mesh to simulate interactions between the organ and the dura mater. It is similar to $\Omega$ except that it satisfies the Signorini conditions $\boldsymbol{\lambda} \perp \boldsymbol{\delta}$ (Duriez et al. 2006). If a vertex, with position $\mathbf{u}_{j}$, is located inside the dura mater $\left(\boldsymbol{\delta}_{j} \geq 0\right)$, no constraint force is applied and $\boldsymbol{\lambda}_{j}=0$. Otherwise, a negative constraint force $\left(\boldsymbol{\lambda}_{j}<0\right)$ is used to cancel the penetration $\left(\overline{\boldsymbol{\delta}}_{j}=0\right)$ and to bring back the vertex on the tangential plane given by $\mathbf{n}_{j}$.

At the end of the resolution, the node $j$, with position $\overline{\mathbf{u}}_{j}$, is located on or 


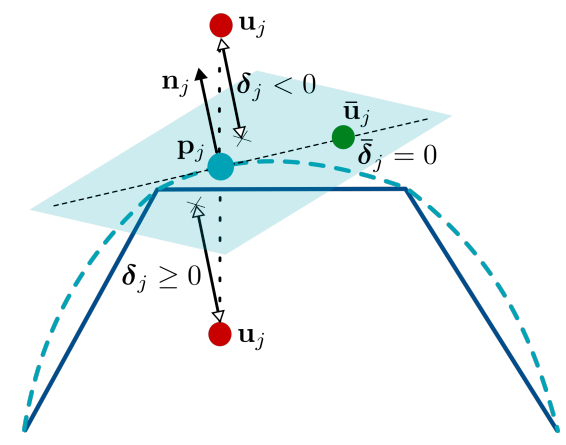

(a) Constraint resolution for $\Omega$

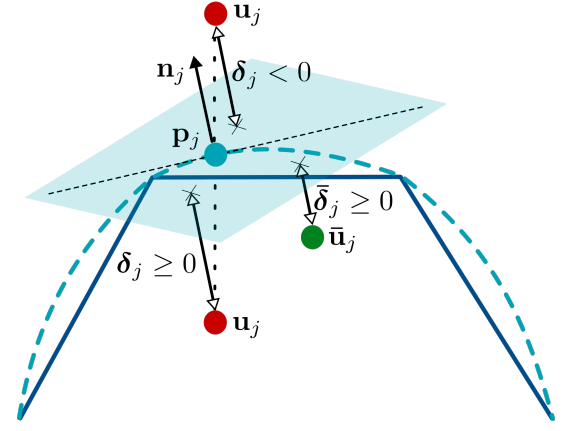

(b) Constraint resolution for $\Psi$

Figure 5: For both figures, the blue broken line and curve are respectively representing the dura mesh and the Bezier surface defined over the triangles of the dura mesh. For $\mathbf{u}_{j}$ (in red), two cases are shown. When $\mathbf{u}_{j}$ is located outside of the dura mesh, the violation is negative $\left(\boldsymbol{\delta}_{j}<0\right)$. Conversely, $\mathbf{u}_{j}$ inside the dura mater corresponds to a positive violation $\left(\boldsymbol{\delta}_{j} \geq 0\right)$. For both cases, this point is projected on $\mathbf{p}_{j}$ (in blue). After the resolution, its position $\overline{\mathbf{u}}_{j}$ (in green) is located on the tangential plane for $\boldsymbol{\Omega}$ and under this plane for $\boldsymbol{\Psi}$.

below this plane (see Figure $5(\mathrm{~b})$. The brain model is thus prohibited to exit it.

\subsubsection{Unilateral probe constraint $\boldsymbol{\beta}$}

$\boldsymbol{\beta}$ is used to impose interactions between the brain surface mesh and the probe footprint point set extracted from intraoperative B-mode US images. Conversely to the dura mesh used for $\boldsymbol{\Omega}$ and $\boldsymbol{\Psi}$, the probe footprint is represented using a point set without any connectivity (edges, triangles, etc.). Projections in the collision detection phase are thus inverted. Each footprint point, with position $\mathbf{p}_{j}$, is associated with $\mathbf{q}_{j}$, its nearest projection over the triangles of the brain surface collision mesh. $\mathbf{q}_{j}$ can then be described using barycentric coefficients computed over the vertices positions $\mathbf{u}_{j_{a}}, \mathbf{u}_{j_{b}}$ and $\mathbf{u}_{j_{c}}$ of its corresponding triangle $j_{a} j_{b} j_{c}$.

The violation of constraint is computed as:

$$
\boldsymbol{\delta}_{j}=\mathbf{n}_{j} \cdot\left(\mathbf{p}_{j}-\mathbf{q}_{j}\right)
$$


with $\mathbf{n}_{j}$ being the normalized normal of the triangle $j_{a} j_{b} j_{c}$. As for $\boldsymbol{\Psi}$, the Signorini conditions are satisfied for $\boldsymbol{\beta}$. The resulting constraint force $\boldsymbol{\lambda}_{j}$ is applied along $\mathbf{n}_{j}$ and distributed on the three vertices $j_{a}, j_{b}$ and $j_{c}$, according to the barycentric coefficients of $\mathbf{q}_{j}$.

After the resolution, these vertices have moved to positions $\overline{\mathbf{u}}_{j_{a}}, \overline{\mathbf{u}}_{j_{b}}$ and $\overline{\mathbf{u}}_{j_{c}}$, such as $\mathbf{p}_{j}$ is located on or above the plane that they form. The brain surface mesh is then situated under the probe footprint point set, but the two are not necessarily in contact. Indeed, during the US acquisition, the probe might not be perfectly in contact with the exposed cortical surface and a gap can appear in the images between the soft tissues and the probe footprint.

\subsubsection{Bilateral vessels constraint $\boldsymbol{\Phi}$}

This constraint is used to register the skeleton of the preoperative vascular tree $\boldsymbol{\zeta}_{M R A}$ with the one extracted from the intraoperative Doppler US images

${ }_{635} \boldsymbol{\zeta}_{U S}$. As $\boldsymbol{\zeta}_{U S}$ is more sparse than $\boldsymbol{\zeta}_{M R A}$, this skeleton is projected towards $\boldsymbol{\zeta}_{M R A}$ for the registration. Each point of $\boldsymbol{\zeta}_{U S}$, with position $\mathbf{p}_{j}$, is associated with $\mathbf{q}_{j}$, being the projection of $\mathbf{p}_{j}$ onto its nearest segment of $\boldsymbol{\zeta}_{M R A} \cdot \mathbf{q}_{j}$ can then be defined from the two extremities of this segment, with positions $\mathbf{u}_{j_{a}}$ and $\mathbf{u}_{j_{b}}$, using barycentric coefficients.

The violation of constraint is computed such as:

$$
\boldsymbol{\delta}_{j}=\left\{\begin{array}{l}
\boldsymbol{\delta}_{j_{1}} \\
\boldsymbol{\delta}_{j_{2}}
\end{array}=\left\{\begin{array}{l}
\mathbf{n}_{j_{1}} \cdot\left(\mathbf{p}_{j}-\mathbf{q}_{j}\right) \\
\mathbf{n}_{j_{2}} \cdot\left(\mathbf{p}_{j}-\mathbf{q}_{j}\right)
\end{array}\right.\right.
$$

640 $j_{a} j_{b}$. The constraint forces $\boldsymbol{\lambda}_{j_{1}}$ and $\boldsymbol{\lambda}_{j_{2}}$ are then computed such that all penetrations are canceled $\left(\overline{\boldsymbol{\delta}}_{j_{1}}=\overline{\boldsymbol{\delta}}_{j_{2}}=0\right) . \quad \boldsymbol{\lambda}_{j_{1}}$ and $\boldsymbol{\lambda}_{j_{2}}$ are respectively applied along $\mathbf{n}_{j_{1}}$ and $\mathbf{n}_{j_{2}}$ and distributed on the vertices $j_{a}$ and $j_{b}$ according to the barycentric coordinates of $\mathbf{q}_{j}$.

645

At the end of the resolution, $\boldsymbol{\zeta}_{M R A}$ has deformed so that $\mathbf{p}_{j}$ is located on the line engendered by the vertices $j_{a}$ and $j_{b}$, with positions $\overline{\mathbf{u}}_{j_{a}}$ and $\overline{\mathbf{u}}_{j_{b}}$ (see Figure 6). 


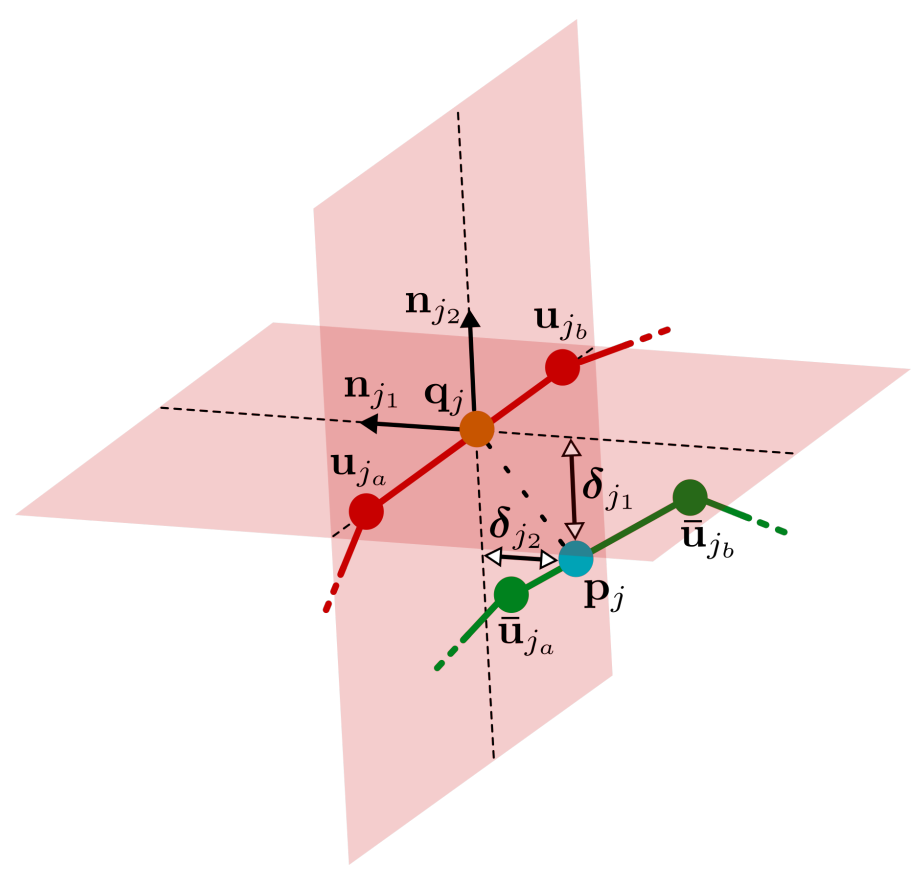

Figure 6: Constraint resolution for $\boldsymbol{\Phi}$. The red and green broken lines corresponds to the position of $\boldsymbol{\zeta}_{M R A}$ before and after the resolution respectively. The point from $\boldsymbol{\zeta}_{U S}$ (in blue), with position $\mathbf{p}_{j}$ is associated with its closest projection $\mathbf{q}_{j}$ (in orange) among the $\boldsymbol{\zeta}_{M R A}$ segments. At the end of the resolution, forces are applied on $\mathbf{u}_{j_{a}}$ and $\mathbf{u}_{j_{b}}$ such as the two violations $\boldsymbol{\delta}_{j_{1}}$ and $\boldsymbol{\delta}_{j_{2}}$ are canceled and $\mathbf{p}_{j}$ is located on the line formed by $\overline{\mathbf{u}}_{j_{a}}$ and $\overline{\mathbf{u}}_{j_{b}}$. 


\subsubsection{Outliers filtering for $\boldsymbol{\Phi}$}

The quality of the vessels registration strongly depends on the quality of the pairings (association of $\mathbf{p}_{j}$ with $\mathbf{q}_{j}$ ) performed during the collision detection of $\mathbf{\Phi}$. However, incoherent or antagonistic pairings (called outliers) can appear due to the noise (image artefact, missing data, etc.) contained in skeletons. These outliers have then to be ignored in order to make our elastic registration algorithm robust.

For this, an outliers filtering step is first added after the collision detection phase of $\boldsymbol{\Phi}$. For all pairings, the distance between a vertex of $\boldsymbol{\zeta}_{U S}$ and its corresponding segment in $\boldsymbol{\zeta}_{M R A}$ is defined as $\mathbf{d}_{j}=\left\|\mathbf{p}_{j}-\mathbf{q}_{j}\right\|$. When several vertices of $\boldsymbol{\zeta}_{U S}$ are projected onto the same segment of $\boldsymbol{\zeta}_{M R A}$, only the nearest one is kept. In addition, a pairing is considered as an outlier, and then ignored, if it does not satisfy the following constraint:

$$
\mathbf{d}_{j} \in\left[\tilde{\mathbf{d}}-\mathbf{d}_{t} ; \tilde{\mathbf{d}}+\mathbf{d}_{t}\right]
$$

where $\tilde{\mathbf{d}}$ is the median distance computed over all pairings and $\mathbf{d}_{t}$, a threshold. For example, if a vessel appears in $\boldsymbol{\zeta}_{U S}$ and not in $\boldsymbol{\zeta}_{M R A}$, incoherent parings might be created with another distant vessel. Such incoherent pairings will then be ignored using the outliers filtering step described above.

Furthermore, during the resolution phase of $\boldsymbol{\Phi}$, the constraint forces $\boldsymbol{\lambda}_{j_{1}}$ and $\boldsymbol{\lambda}_{j_{2}}$ are computed such as:

$$
\left(\boldsymbol{\lambda}_{j_{1}}, \boldsymbol{\lambda}_{j_{2}}\right) \in\left[-\mathbf{f}_{\max } ; \mathbf{f}_{\max }\right]^{2}
$$

with $\mathbf{f}_{\max }$ a parameter defining the maximal force applied on the vessels. Since the constraint forces are bounded, all constraints might not be satisfied at the end of the resolution $\left(\left(\overline{\boldsymbol{\delta}}_{j_{1}}, \overline{\boldsymbol{\delta}}_{j_{2}}\right) \neq(0,0)\right)$. When vessels are close or skeletons are noisy, a segment of $\boldsymbol{\zeta}_{M R A}$ may be associated with a vertex of $\boldsymbol{\zeta}_{U S}$ in a different direction than its neighbors. In such a case, huge constraint forces will be needed to satisfy these pairings, which might destabilized the simulation.

${ }_{665}$ Bounding the constraint forces thus enables the simulation convergence even if some outliers pairings remain. In addition, each constraint response $\left(\boldsymbol{\lambda}_{j_{1}}\right.$ and 
$\boldsymbol{\lambda}_{j_{2}}$ ) being individually bounded, the total registration force applied on the brain model depends on the number of constraints. However, since the US skeleton is decimated, the local constraint number is controlled, guaranteeing that no huge force is applied locally.

Finally, the registration constraint parameters $\mathbf{d}_{t}$ and $\mathbf{f}_{\max }$, defined in the above paragraphs, are set to $1.5 \mathrm{~mm}$ and $0.001 \mathrm{~N}$ respectively. These ad hoc values were optimized over the first clinical case and remain the same for all other simulations.

\subsection{Update of preoperative images}

After running the constraint-based biomechanical simulation, the brain model has deformed, registering the blood vessels and constraining the cortical surface (see Figures 7(a) and 7(b) . Preoperative MR images have thus to be updated to account for this simulated brain-shift deformation. A displacement field is first computed on the entire MR volume based on the deformations of the FE mesh, combined with barycentric coefficients. Preoperative images are then warped with the previous displacement field to create navigable intraoperative MR images, that now correspond to the current patient anatomy. As highlighted by (Li et al. 2014), warping a set of images is a complex problem. In this paper, this process is based on linear interpolations to determine the voxels' grey levels, as implemented in the VTK library (Schroeder et al. 2006 ). Preoperative and updated MR images are shown in Figures 7(c) and 7(d) respectively.

\section{Experiments}

The experiments performed on our method are introduced in this section. First, details about the implementation are provided. The generation of synthetic data is then described before presenting the five surgical cases.

\subsection{Implementation}

For each clinical case, brain tissues were segmented from T2-FLAIR MR images. The BET tool available in the FSL software (Smith, 2002) was used for 


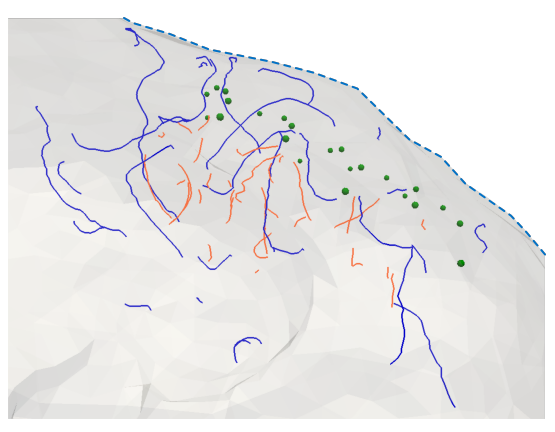

(a) Brain model before compensation

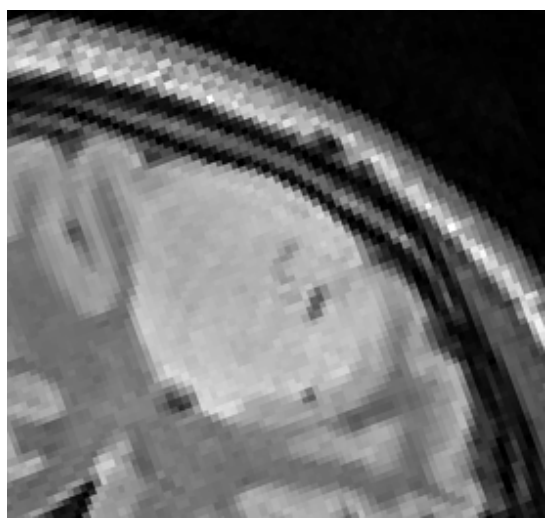

(c) Preoperative MRI

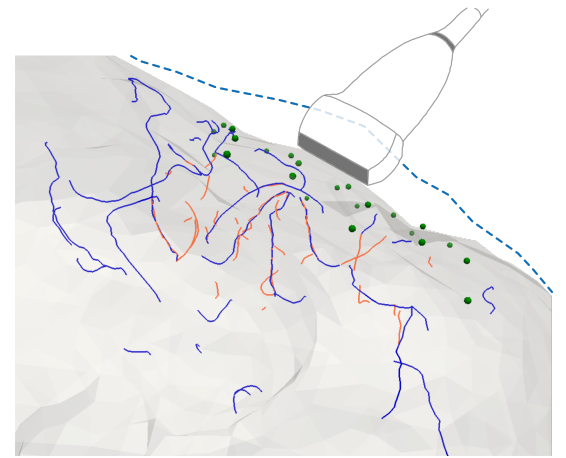

(b) Brain model after compensation

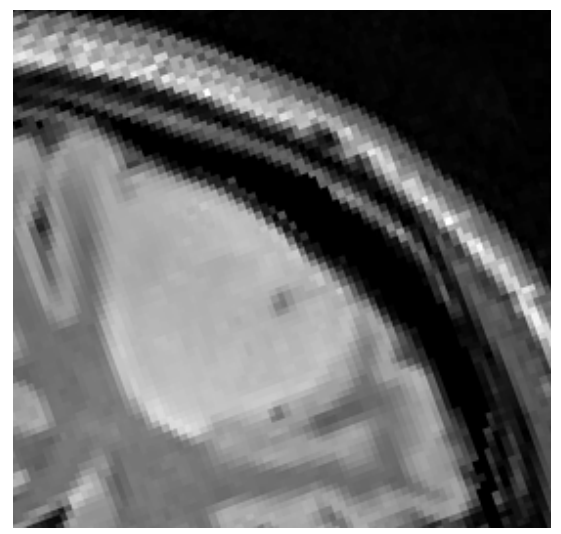

(d) Updated preoperative MRI

Figure 7: Brain-shift compensation using our constraint-based method and update of preoperative images. For the brain models, blood vessels extracted from the preoperative MRA and intraoperative Doppler US images are respectively displayed in blue and orange. The probe footprint point set coming from the B-mode US acquisitions is shown in green. 

SNAP (Yushkevich et al., 2006). The FE meshes were then created with CGAL (CGAL, 2016). All others steps, including the definition of the boundary conditions, the segmentation and skeletonization of the vessels and the extraction of the probe footprint, were implemented as plug-ins within the visualization developed using the simulation framework Sofa (Faure et al. 2012).

As detailed above, several software frameworks were used to develop our method, limiting a practical use in a clinical environment. However, the main objective of this paper is to provide a proof of concept of a new method that (i.e. same meshes, constitutive laws and parameters, boundary conditions, etc.), the registration results should only depend on the input data (US skeleton and probe footprint) and constraint parameters. 
Table 1: Mean distances \pm std between vessels and tumor landmarks after the generation of synthetic deformation. Maximum distances are given in parenthesis. All distances are provided in $\mathrm{mm}$.

\begin{tabular}{ccc}
\hline Landmarks type & Number of landmarks & Distance \\
\hline Vessels landmarks & 7 & $4.27 \pm 1.18(6.49)$ \\
Tumor landmarks & 33 & $5.94 \pm 1.04(7.77)$ \\
\hline
\end{tabular}

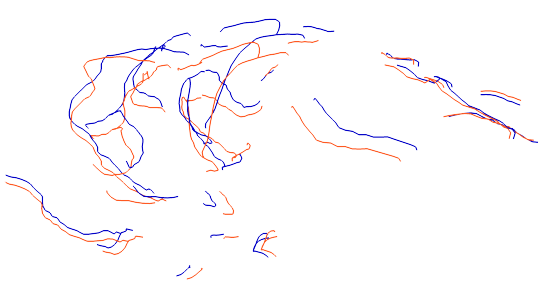

(a) Generated skeleton $\boldsymbol{\zeta}_{\text {generated }}$

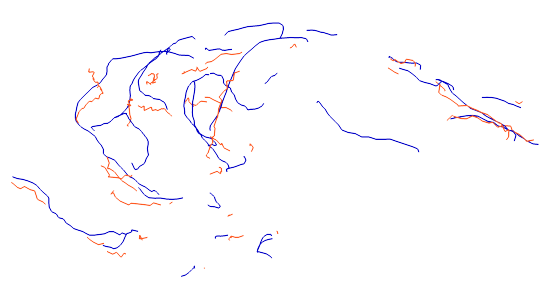

(c) Sparse skeleton $\boldsymbol{\zeta}_{\text {sparse }}(50 \%$ of points removed)

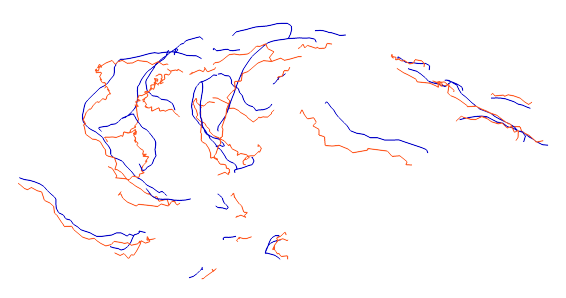

(b) Perturbed skeleton $\boldsymbol{\zeta}_{\text {perturbed }}$

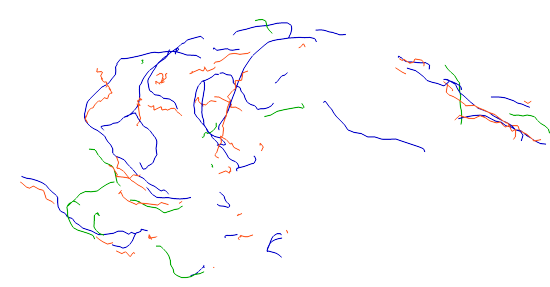

(d) Final synthetic skeleton $\boldsymbol{\zeta}_{\text {synthetic }}(50 \%$ of points added, in green)

Figure 8: Generation of a synthetic skeleton (orange) compared to the initial skeleton (blue) 


\subsubsection{Generation of a brain-shift deformation}

A biomechanical model, built from the preoperative MR images of clinical case 1 (see Section 7.3), is used to generate the deformation. Arbitrary forces are applied on the brain surface, close to the craniotomy region, and around the tumor in order to simulate a brain-shift deformation (see Figure 8(a) . This deformation is then obtained using meaningless applied forces, simplified behavior models and boundary conditions and does not account for registration errors between preoperative MRI and intraoperative US images. Therefore, it cannot correspond to a physically plausible deformation obtained under real clinical conditions. However, the resulting displacements (see Table 1) can be compared to the ones observed in surgical cases (see Table 5). While it is not sufficient to assess the accuracy of the complete brain-shift compensation method, this deformation is nevertheless used to evaluate the robustness of its ad-hoc parameters. The deformed blood vessels skeleton $\boldsymbol{\zeta}_{\text {generated }}$ and brain surface mesh are then extracted. Finally, the brain surface mesh is cropped to the craniotomy region in order to represent the probe footprint that could be extracted from B-mode US images.

The deformation is quantified using 40 landmarks distributed on the vascular tree and on the tumor surface, summarized in Table1. Seven landmarks are set on the blood vessels and located on the bifurcations of the skeleton, similarly to the ones used in Section 7.3.1 to validate our method on clinical cases. The other landmarks are uniformly placed on the tumor surface in order to monitor the impact of the registration method on the surgical target.

\subsubsection{Addition of noise}

For real clinical cases, only noisy skeletons, partially corresponding to the preoperative MR one, are extracted from intraoperative US images. This noise has two main sources: the data themselves and their segmentation. As we treat images from very different modalities and qualities, a vessel can be visible in MRA and not in US acquisitions (e.g. vessels in the direction of the US wave propagation) and vice versa (e.g. US acquisition noise and/or 3D reconstruction 


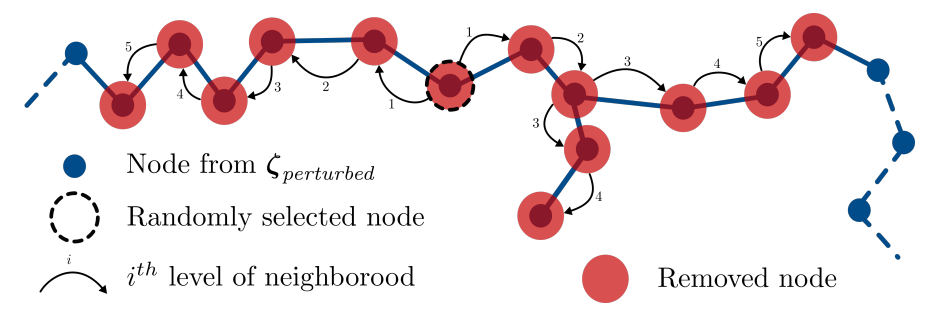

(a) Nodes removal

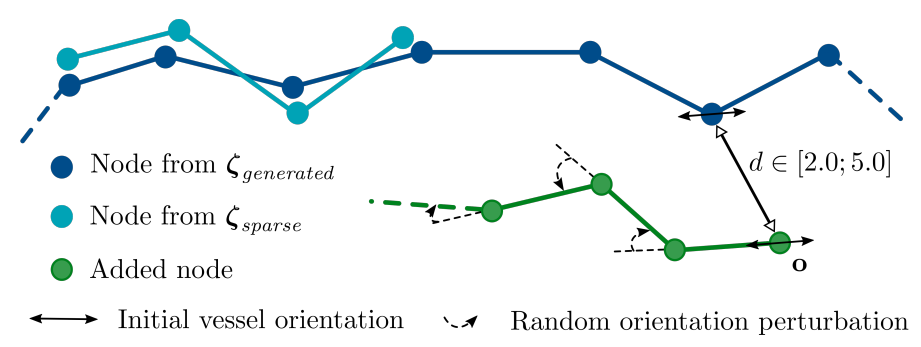

(b) Vessels addition

Figure 9: Addition of noise in synthetic skeletons

Nodes removal. Iteratively, a node is randomly selected and removed from $\boldsymbol{\zeta}_{\text {perturbed }}$. However, in order to remove pieces of branch and not only isolated points, its first to fifth level neighbors are also deleted, as shown in Figure 9(a) 
The sparsity of the output skeleton is controlled by removing a given percentage of nodes from $\boldsymbol{\zeta}_{\text {perturbed }}$. For example, $50 \%$ of points were deleted in the skeleton presented in Figure 8(b) to obtain the sparse skeleton $\boldsymbol{\zeta}_{\text {sparse }}$ of Figure 8(c).

Vessels addition. Extra vessels are added to $\boldsymbol{\zeta}_{\text {sparse }}$. A new vessel is defined by an origin $\mathbf{o}$, with coordinates randomly chosen in the 3D space, a random number of points $N$ and an orientation vector $\mathbf{v}$. In order to maximize the probability of erroneous pairings during the registration process, the distance between $\mathbf{o}$ and its closest point in $\boldsymbol{\zeta}_{\text {generated }}$ is included between 2.0 and 5.0 $\mathrm{mm}$. In addition, $\mathbf{v}$ is initialized parallel to the closest vessel with a random perturbation added at each iteration. Finally, the vessel is built point per point from its origin $\mathbf{o}$, until $N$ is reached:

$$
\mathbf{p}_{\mathbf{i}}=\mathbf{p}_{\mathbf{i}-\mathbf{1}}+\mathbf{v}_{\mathbf{i}-\mathbf{1}}, \quad \forall i \in\{1, . ., N-1\}, \quad \mathbf{p}_{\mathbf{0}}=\mathbf{o} \quad \text { and } \quad \mathbf{v}_{\mathbf{0}}=\mathbf{v}
$$

with $\mathbf{p}_{\mathbf{i}}$ and $\mathbf{v}_{\mathbf{i}}$ respectively the built position and normalized orientation vector at iteration $i$ (see Figure 9(b) for details). The total number of added points (combining all extra vessels) is set as a percentage of the initial number of points of $\boldsymbol{\zeta}_{\text {sparse }}$. The final synthetic skeleton $\boldsymbol{\zeta}_{\text {synthetic }}$ shown in Figure $8(\mathrm{~d})$ was obtained by adding $50 \%$ of points to the sparse one presented in Figure $8(\mathrm{c})$

Even with a segmentation threshold set manually, the acquisition and extraction noises observed for the probe footprint are far less important than for the US skeleton. However, some reconstruction artifacts can result on a misalignment of few millimeters between two consecutive US slices and then irregularities on the point set extracted as the probe footprint. Therefore, only the $3 \mathrm{D}$ perturbation detailed above is added to the coordinates of each point of the generated probe footprint. Correlated to the size of the reconstruction artifacts, the perturbation interval is also set to $[-0.5 ; 0.5]$ (in $\mathrm{mm}$ ).

Finally, the synthetic skeleton and probe footprint are prepared for the simulation. As described in Section 5.2.3, these data are decimated and contacts with the dura mater at the craniotomy location are deactivated. 
Table 2: Clinical cases, all of them operated for a low-grade tumor. For the validation, the segmented anatomical structure and the number of landmarks identified on blood vessels are given for each case.

\begin{tabular}{cccc}
\hline Patient & Location & Vessels landmarks & B mode structures \\
\hline 1 & Left frontal lobe & 12 & Prefrontal sulcus \\
2 & Insula and left temporal lobe & 14 & Sylvian fissure \\
3 & Insula and right temporal lobe & 16 & Right ventricle \\
4 & Right insula & 17 & Superior temporal sulcus \\
5 & Left prefrontal lobe & - & Cortical sulcus \\
\hline
\end{tabular}

\subsection{Patient data}

In this study, five patients suffering from a low-grade tumor are included.

They underwent brain tumor surgery at Saint Olav University Hospital (Trondheim, Norway). Data were collected by the SINTEF Medical Technology (Trondheim, Norway) with Sonowand Invite (Sonowand AS, Trondheim, Norway). The study was approved by the local ethics committee, and patients gave informed consent prior to the procedure.

The protocol is similar to the one followed by Reinertsen et al. (2014). For each case, MR scans (T2-FLAIR and Angiography) were acquired before surgery. Intraoperatively, US acquisitions were performed for navigation as well as to monitor the soft tissues and vessels deformations. The clinical cases are presented in Table 2 .

For the validation of our compensation method, the warped preoperative MRI must be compared with actual intraoperative information. While objective methods exist in the literature (Garlapati et al., 2015), they require data of the same modality (intraoperative MRI in that case). In our context, US images are the only intraoperative information available. Hence, a more subjective and potentially less-repeatable validation process had to be used. Two quantitative analysis are thus proposed, on vessel landmarks then on soft tissues structures. 


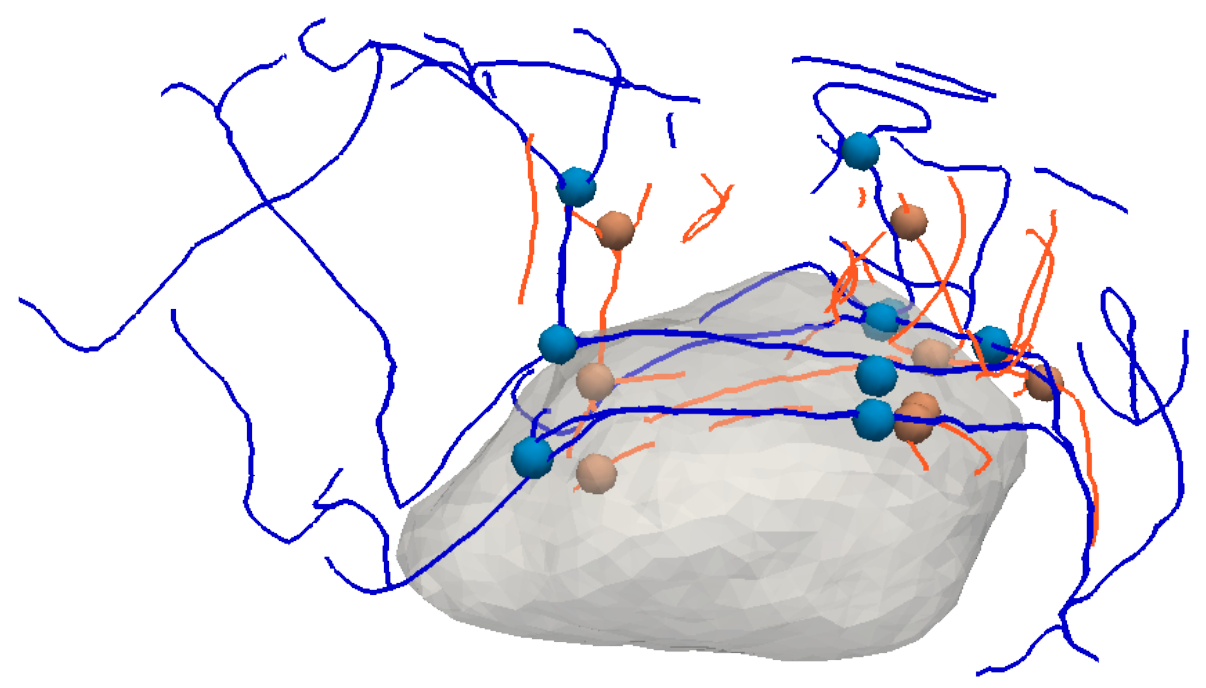

(a) Paired landmarks identified by the second operator on the pre-operative (blue) and intraoperative (orange) blood vessels. Even if the landmarks are directly identified on the medical images, the vessels are shown in this figure as skeletons for clarity.

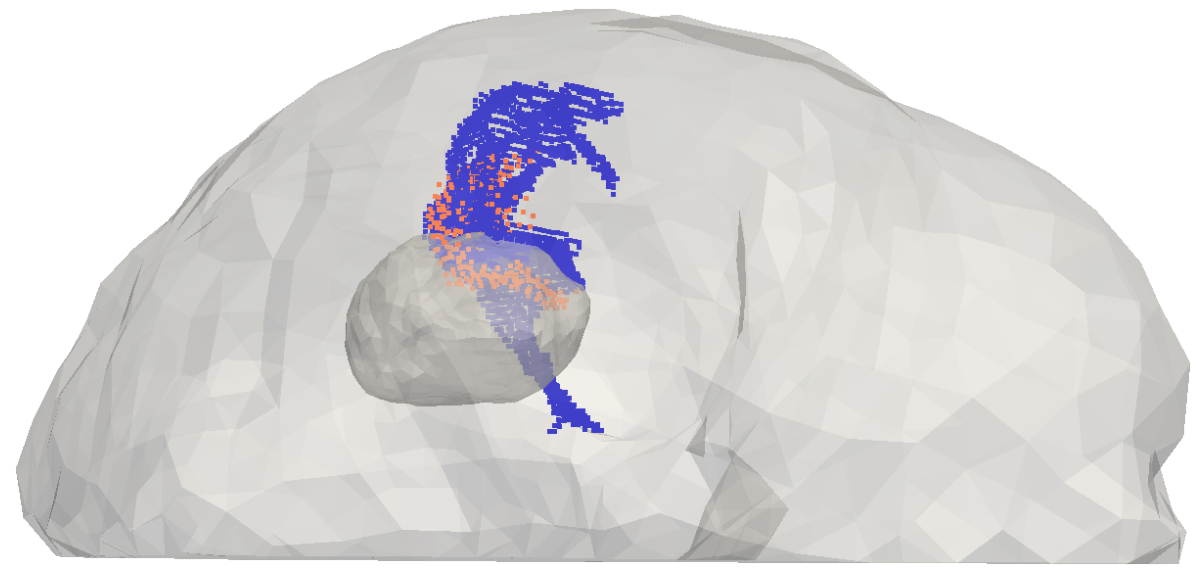

(b) Sets of points representing the superior temporal sulcus segmented in the pre-operative MRI (blue) and intra-operative B-mode US (orange)

Figure 10: Example of validation data for clinical case 4 


\subsubsection{Identification of vessels landmarks}

A few landmarks were manually set on two vessels datasets: the one extracted from MR images and the one segmented from intra-operative Power Doppler US images. Depending on the data, 5 to 9 landmarks are identified for each patient by two operators. Salient features are looked for, like bifurcations or high curvature points (see Figure 10(a). For patient 5, no landmarks could be set due to the lack of reliable vessel structures (very sparse signal of poor quality) in the region of interest. The registration accuracy is then measured by computing distances between paired vessels landmarks before and after compensation.

a Although this process is common in the literature and followed by Reinertsen et al. (2014), two limitations could be pointed out. First, a bias exists when measuring errors on vessels while these same vessels are used for the registration although they are not treated similarly. Next, positioning landmarks on vessels with high accuracy is quite difficult, especially on US data. In addition, this manual process is subjective and probably non repeatable. To account for these drawbacks, landmarks positioning was realized twice for each patient by two different users. Average and maximum distances between paired vessels are displayed in Table 3 . Even if obtained values are in the same ranges, some inter-operator differences are also shown. These differences are obviously explained by the difficulty and subjectivity of the process. However, since it was achieved blindly (i.e. an operator does not know the positioning of the other operator's landmarks), landmarks were not set on the same salient features by the two operators, thus implying variations in the obtained distances. Finally, our method is evaluating based on the reunification of these two landmarks sets for each clinical case. Such data thus provide higher variability in the positioning of landmarks (location and repartition) and account for the subjectivity of the process. 
Table 3: Mean distances \pm sd between paired vessels landmarks blindly identified by two operator then reunified in a unique dataset (synthesis). Maximum distances are given in parenthesis. All distances are provided in $\mathrm{mm}$.

\begin{tabular}{ccccccc}
\hline \multirow{2}{*}{ Patient } & \multicolumn{2}{c}{ Operator 1 } & \multicolumn{2}{c}{ Operator 2 } & \multicolumn{2}{c}{ Synthesis } \\
& number & distances & number & distances & number & distances \\
\hline 1 & 7 & $3.97 \pm 1.30(5.86)$ & 5 & $4.49 \pm 1.02(5.79)$ & 12 & $4.19 \pm 1.22(5.86)$ \\
2 & 5 & $3.56 \pm 0.27(3.95)$ & 9 & $3.48 \pm 0.86(4.77)$ & 14 & $3.51 \pm 0.71(4.77)$ \\
3 & 7 & $7.67 \pm 1.19(9.04)$ & 9 & $7.06 \pm 1.23(8.97)$ & 16 & $7.32 \pm 1.25(9.04)$ \\
4 & 8 & $6.27 \pm 0.67(7.02)$ & 9 & $6.41 \pm 0.63(7.45)$ & 17 & $6.35 \pm 0.65(7.45)$ \\
5 & - & - & - & - & - & -
\end{tabular}
images while being completely delineated within MR slices. As a result, huge putliers can appear that even robust versions of Hausdorff distance (Fedorov 
et al. 2008) are not able to treat. That's why a more global metric, translating the average registration score, is used to quantify the brain-shift compensation. The mean closest-point Euclidean distance is then computed before and after registration, similarly to Clements et al. (2016). However this measurement is a minimization of the actual error: the closest neighbor of a given point is not necessarily its adequate match in the other surface. Hence, if two points would be associated to the same correspondent, only the closest pair is kept. The total number of associated points gives an indication of the pairing quality (100\% meaning each US point has a single pair in the MR contour, which would corresponds to a quasi-perfect superposition).

\section{Results}

In this section, quantitative and qualitative results are provided to evaluate our method. While the first paragraphs focus on its compatibility with a surgical process, its efficiency to compensate for brain-shift is shown in the next sections, first over synthetic data and finally over patient data.

\subsection{Integration to the surgical pipeline}

In order to be compatible with a surgical process, a method should be as automatic as possible, limiting the user interactions, and clinically relevant in terms of execution times.

\subsubsection{User interactions}

Limiting the user interactions, especially during the intervention, is a predominant criterion in order to be compatible with a surgical process. Our method was thus developed so that most of the steps, and mainly the intraoperative ones, are automatically executed. However, it is not fully automatic and some steps still require interactions with an operator.

Our method does not need a detailed soft tissues segmentation of the preoperative MRI: only the envelopes of the brain, cerebellum, tumor and the falx cerebri are needed. For the clinical cases presented in Section 7.3 , the 
Table 4: Summary of the parameters, their types, uses, values and how they are set

\begin{tabular}{|c|c|c|c|c|}
\hline Type & Section & Parameter/Name & Value & Configuration \\
\hline \multirow{2}{*}{ Data extraction } & \begin{tabular}{|l|}
5.2 .1 \\
5.2 .2 \\
\end{tabular} & $\begin{array}{l}\text { Vessels segmentation threshold } \\
\text { Footprint segmentation threshold }\end{array}$ & - & Fixed by an operator \\
\hline & 5.2 .3 & $\begin{array}{l}\text { Vessels decimation threshold } \\
\text { Footprint decimation threshold }\end{array}$ & $\begin{array}{l}2.5 \mathrm{~mm} \\
10 \mathrm{~mm} \\
\end{array}$ & $\begin{array}{l}\text { Set regarding } \\
\text { meshes resolution }\end{array}$ \\
\hline Biomechanical & 6.1 & $\begin{array}{c}\text { Young's modulus } E \\
\text { Tumor Young's modulus } \\
\text { Poisson's ratio } \nu\end{array}$ & $\begin{array}{c}1.5 \mathrm{kPa} \\
10 \mathrm{kPa} \\
0.45\end{array}$ & Taken from the literature \\
\hline $\begin{array}{l}\text { Registration } \\
\text { constraint }\end{array}$ & 6.2 .5 & $\begin{array}{l}\text { Outliers median threshold } \mathbf{d}_{t} \\
\text { Maximal registration force } \mathbf{f}_{\max }\end{array}$ & $\begin{array}{l}1.5 \mathrm{~mm} \\
0.001 \mathrm{~N}\end{array}$ & Optimized over a first patient \\
\hline
\end{tabular}

segmentation of some brain anatomical structures was realized by an operator (see Section 5.1.1 for the details), which is not a satisfactory solution in a surgical process. However, this problem is fundamental in medical simulation and has been widely addressed in the literature. For example, automatic atlas-based segmentation algorithms experimented on brain MRI were recently presented at the MICCAI'2016 conference and workshops (Shakeri et al., 2016, Benkarim et al., 2016, Arthofer et al., 2016). The obtained brain segmentations are then much more detailed and accurate than the ones needed for our method. Finally, although this is not the topic of this paper, some solutions exist in the literature in order to automatically extract the brain tissues and anatomical structures.

Moreover, our method still requires several parameters, summarized in Table 4 Even if most of them are fixed for all simulation cases, the segmentation thresholds for the vessels and the probe footprint extracted from the intraoperative US images have to be manually set by an operator for each patient. These thresholds depend on the quality of the input US image but also of the US scanner settings selected by the surgeon (gain, etc.). Depending on the operator, the chosen thresholds may thus be different, leading to data with various levels of noise. However, since these data (the intra-operative vessels and probe 


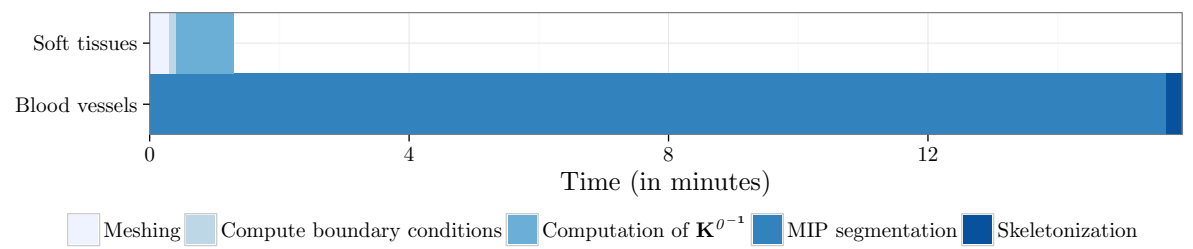

(a) Pre-operative execution times. For building the brain model, the segmentation time is not accounted for.

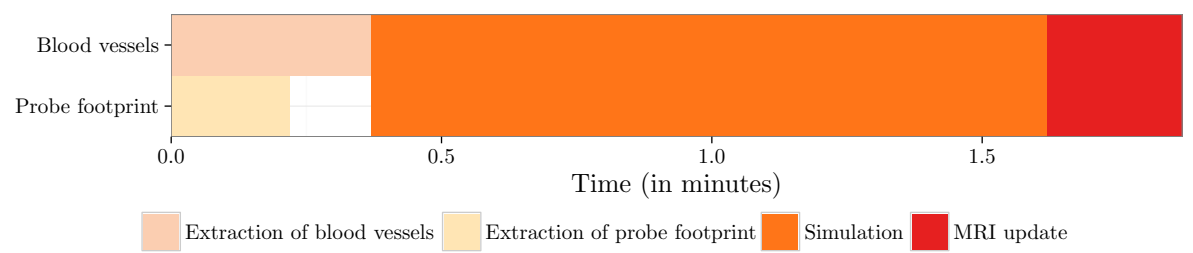

(b) Intra-operative execution times, after US acquisition and 3D volume reconstruction.

Figure 11: Execution times of each step of our method

900 could have a strong impact on our method and its parameters, especially the parameters set for the registration constraint. The robustness of our method to these parameters is evaluated over synthetic data in Section 8.2

\subsubsection{Execution times}

905 times is another essential criterion. The pre- and intraoperative execution times of each step are respectively presented in Figure 11(a) and 11(b).

Since MR brain images are usually acquired one day prior to surgery, several hours are available to process the preoperative data. Using our method, the brain $\mathrm{FE}$ and surface meshes can be built simultaneously as the blood vessels extraction from MRA. As shown in Figure 11(a) all these steps are executed in less than 16 minutes and are thus completely compatible with a clinical workflow. However, we assumed that the semi-automatic soft tissues segmentation, that currently needs approximately one hour and occurs before the meshing 915 step, could be processed with a method from the literature (discussed in Sec- 


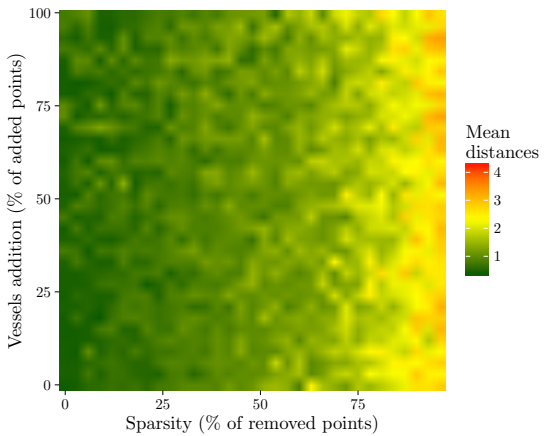

(a) Results on vessels landmarks

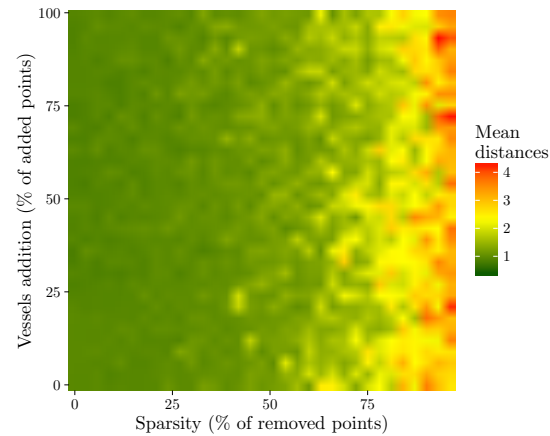

(b) Results on tumor landmarks

Figure 12: Validation on synthetic data. For each configuration, the median mean landmarks distance over three synthetic skeletons is displayed.

tion 8.1.1). Only our additional computing time is therefore evaluated in Figure 11(a). As a result, if a time relevant solution exists in the literature for segmenting soft brain tissues, our method will also be time relevant.

口 Intraoperative execution times are presented in Figure 11(b), Reinertsen et al. (2014) already showed that performing US acquisition and 3D volume reconstruction during brain surgery is time relevant and thus, only our additional executions times are evaluated. Since the blood vessels and probe footprint are extracted from the US images in parallel, less than 2 minutes are necessary to simulate the brain deformations and obtain the updated preoperative MRI. In conclusion, this additional intraoperative time is acceptable considering the fact that a typical brain tumor resection procedure lasts several hours.

\subsection{Validation on synthetic data}

The efficiency and robustness of our method, and more specifically of its ad-hoc registration parameters (see Table 4), was first assessed over synthetic data sets. US skeletons and probe footprint were thus created using the algorithms described in Section 7.2. Deformed skeletons with various levels of sparsity (between 0 and $96 \%$ points removed) and noise (between 0 and $100 \%$ points added) were generated. For each configuration, three skeletons were cre- 
ated. Our constraint-based simulation method was then run for each one of these skeletons, computing the mean distances over the vessels and tumor landmarks. The median of these mean distances is displayed in Figure 12 , for each configuration.

A clear correlation between the results of the vascular tree and tumor surface registrations is shown by Figures 12(a) and 12(b) . When the registration accuracy improves over the vessels, it is also increased for the surgical target. In addition, the method seems more robust to the addition of noise than to the removal of vessels, especially for the tumor registration. Indeed, if a vessel is associated with a wrong neighboring vessel, the distances computed on the vessels landmarks might be highly destabilized. Conversely, deformations of the

945 tumor are interpolated by the biomechanical model from the ones induced to the vascular tree and by the probe footprint. Then, a slight error on the association of the vessels does not significantly disturb the registration results of the tumor.

Finally, as shown in Figure 12, our constraint-based method is efficient to compensate for the generated brain-shift even for configurations where $75 \%$ vessels were removed, whatever the percentage of points added. Beyond this percentage, the method has difficulties to compensate for the deformations but does not diverge: whatever the configuration, the initial mean distances (see Table 1) are never increased. A large range of input data can thus be treated 95 with our method, limiting the impact of the inter-operator differences for the settings of the segmentation thresholds (see Table 4). In addition, if the registration constraint parameters were optimized over the first surgical case, they are robust enough to obtain good registration results for various input skeletons.

\subsection{Validation on patient data}

After studying the method on synthetic data, the next validation step was carried out retrospectively on actual patient images. Two quantitative analysis are proposed on vessel landmarks and then on soft tissue structures. The validation protocols are detailed in Section 7.3 and only the results are pre- 
Table 5: Mean distances \pm sd between paired vessels landmarks before compensation, after rigid registration proposed by Reinertsen et al. (2014) and following our constraint-based registration method. Maximum distances are given in parenthesis. All distances are provided in $\mathrm{mm}$.

\begin{tabular}{cccc}
\hline Patient & Before compensation & Rigid registration & Constraint-based registration \\
\hline 1 & $4.19 \pm 1.22(5.86)$ & $2.29 \pm 1.36(5.25)$ & $1.79 \pm 0.88(3.66)$ \\
2 & $3.51 \pm 0.71(4.77)$ & $1.71 \pm 0.77(3.88)$ & $1.26 \pm 0.18(2.70)$ \\
3 & $7.32 \pm 1.25(9.04)$ & $2.84 \pm 1.27(5.61)$ & $2.33 \pm 0.22(4.85)$ \\
4 & $6.35 \pm 0.65(7.45)$ & $1.91 \pm 1.06(4.37)$ & $1.40 \pm 0.44(3.13)$ \\
5 & - & - & - \\
\hline
\end{tabular}

sented in the following paragraphs. The compensation results obtained with our constraint-based method are compared to the ones obtained with the rigid registration algorithm proposed by Reinertsen et al. (2014) and available in the CustusX navigation tool (Askeland et al., 2015). Finally, a qualitative analysis presents our results with respect to clinical expectations.

\subsubsection{Quantitative evaluation on vessels landmarks}

Distances between paired vessels landmarks are given in Table 5 , before compensation, after rigid registration proposed by Reinertsen et al. (2014) and following our registration method. Figure 13 also shows the repartition of these distances. In every case, the mean error with our process is reduced in comparison to the existing CustusX method. Moreover, the standard deviation is lower and the maximum error is importantly lower. This means our results are more accurate not only globally but for every vessel.

As stated in Section 7.3.1 such evaluation process, based on vessels landmarks, has several drawbacks. However, it is the one followed by Reinertsen et al. (2014), and still shows a quantitative improvement with our method. 980 Especially, accounting for all patient, on average $67 \%$ of the deformation is corrected with our constraint-based method, against $57 \%$ with the rigid one. 


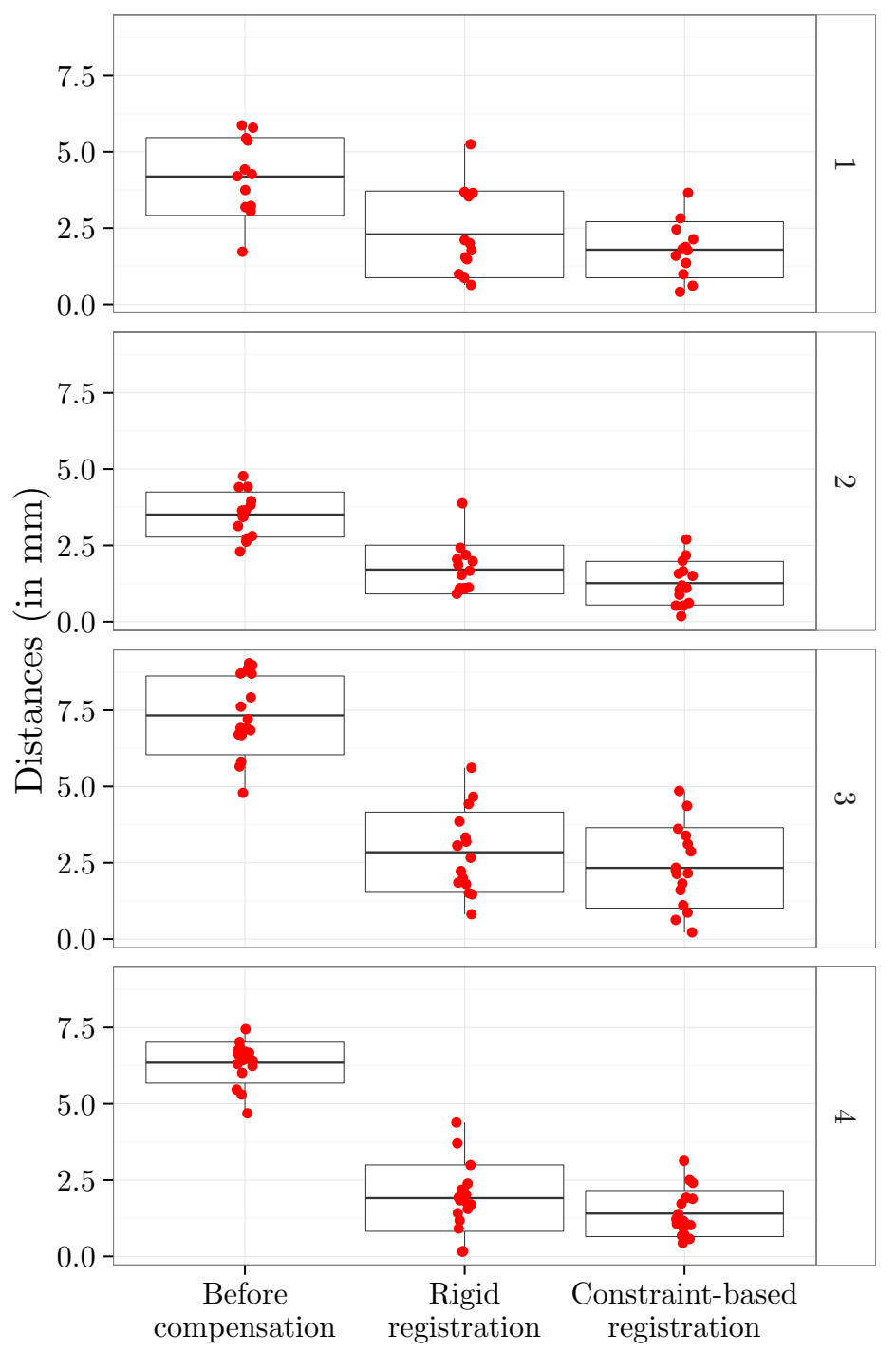

Figure 13: Dispersion of the distances between the paired landmarks defined on the blood vessels, for patients 1 to 4 . While the mean distances \pm std and minimal and maximal distances are shown by the boxes, the distances between each associated landmarks are displayed using red dots. 
Table 6: Mean closest-point Euclidean distances \pm sd between the anatomical structures (represented as point sets) described in Table 2 Maximum closest-point distance is given in parenthesis and the number of associated points is shown in square brackets. These values are provided before compensation, after rigid registration proposed by Reinertsen et al. (2014) and following our constraint-based registration method.

\begin{tabular}{cccc}
\hline Patient & Before compensation & Rigid registration & Constraint-based registration \\
\hline 1 & $1.91 \pm 1.04(6.18)[63 \%]$ & $1.53 \pm 0.92(4.59)[72 \%]$ & $1.03 \pm 0.76(5.17)[90 \%]$ \\
2 & $1.48 \pm 1.07(6.18)[66 \%]$ & $1.28 \pm 0.82(4.66)[79 \%]$ & $1.18 \pm 0.73(4.16)[77 \%]$ \\
3 & $3.86 \pm 2.64(10.93)[42 \%]$ & $1.85 \pm 1.56(6.89)[75 \%]$ & $3.21 \pm 2.72(11.34)[55 \%]$ \\
4 & $2.48 \pm 1.45(5.64)[59 \%]$ & $1.27 \pm 0.82(4.36)[82 \%]$ & $0.98 \pm 0.64(3.90)[86 \%]$ \\
5 & $3.44 \pm 1.76(7.23)[42 \%]$ & $3.04 \pm 3.00(12.34)[12 \%]$ & $2.73 \pm 1.39(6.18)[48 \%]$ \\
\hline
\end{tabular}

\subsubsection{Quantitative evaluation using anatomical structures}

While the measured distances before and after registration with CustusX and our constraint-based method are given in Table 6 , the dispersion of these distances is shown in Figure 14. As can be seen, the registration accuracy is clearly improved for patients 1,2 and 4 . Not only the mean, standarddeviation and maximum errors are reduced, but the number of associated points is increased. That means the error measurement is more reliable, less minimized, with our method.

For patient 3 , the error is only slightly reduced, far less than with the CustusX rigid method. Even if the vessel quantification was improved (see Table 5 ) for this patient, the simulated state is thus not satisfactory. In fact, the probe footprint measured in the US images (supposed to be in contact with the brain) is out of the cranial cavity in the MR images/model. Although tissue could locally and slightly sag out through the craniotomy, this is probably due to a large error of localization during the acquisition process. This error cannot be corrected with our compensation method since the brain model is constrained by the dura surface (see Section 6.2.2). If this constraint was released, results on both the vessels and tissue would be better with our method. This case mostly

stresses the importance of an accurate pre- to intraoperative registration before 

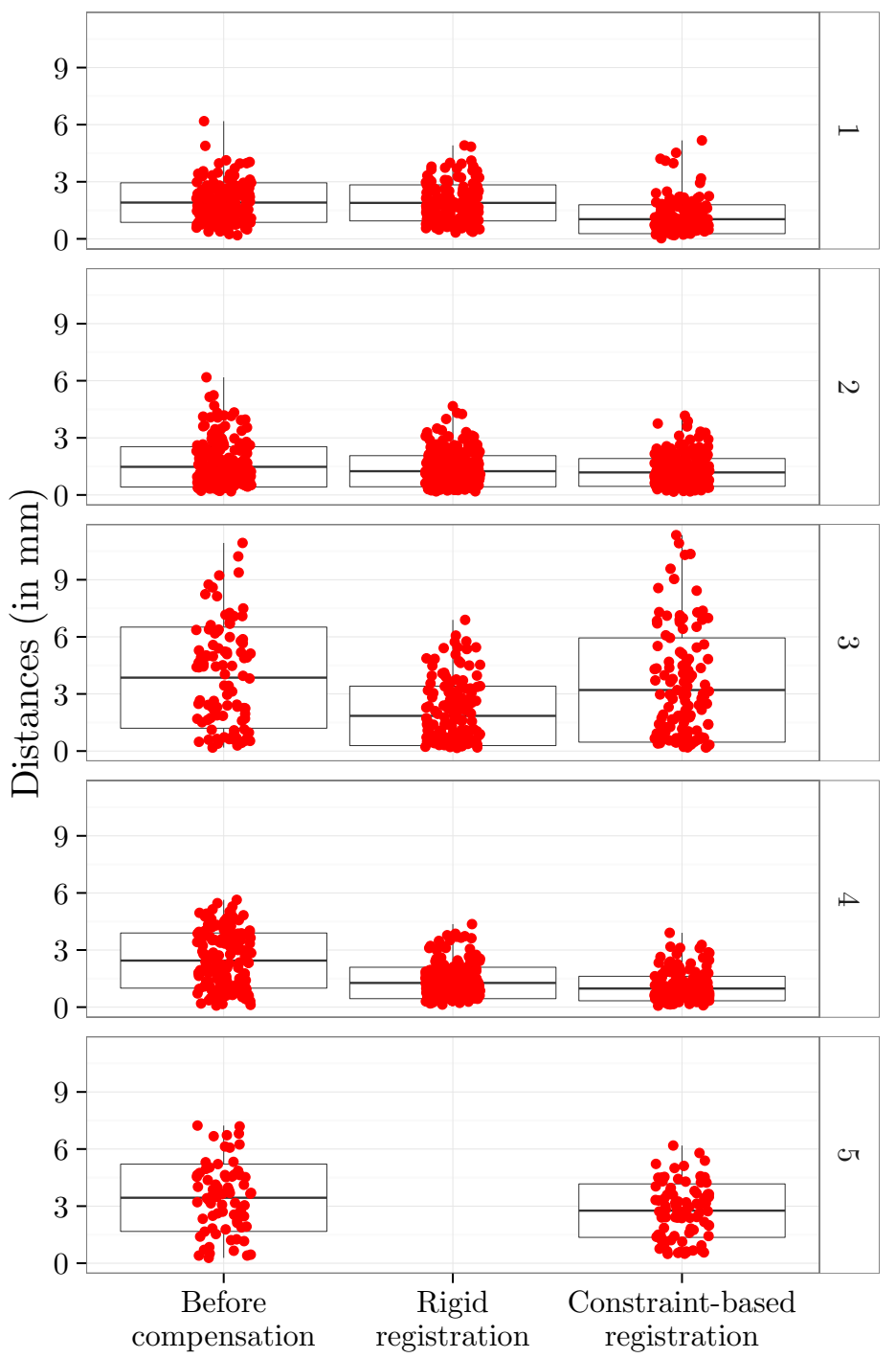

Figure 14: Dispersion of the closest-points distances between the associated anatomical structures delineated in MR and B-mode US images, for patients 1 to 5. While the mean distances \pm std and minimal and maximal distances are shown by the boxes, the distances between each matched point are displayed using red dots. 
acquiring the US images.

Concerning case 5, vessel data are so sparse in the region of interest that it was not possible to identify paired landmarks in the US and MR images. However, both registration methods were still run normally. As can be seen in Table 6 , the rigid method actually fails. Even if the mean error seems acceptable, the maximum distance and number of associated points show that the algorithm diverged. On the other hand, our method still yields a coherent result even if the improvement over the initial position is low. In conclusion, not only our constraint-based method is more accurate but it is also more robust to lowquality sparse data.

\subsubsection{Qualitative results}

Beyond quantitative measurements, results must also be qualitatively evaluated with respect to surgeons expectations, especially the updated MR images that would eventually be used during surgical navigation. In the current CustusX navigation process (Askeland et al. (2015)), the initial MR images are displayed next to the intraoperative US images. In addition to these views, Figure 15 also shows the MR images updated with the computed deformation field for patients 1,2 and 4. For each clinical case, a 3D view of the brain and tumor is presented, highlighting the 3D localization of the surgical target within the organ.

Two points are localized in the medical images, first on the exposed cortical surface then in subsurface structures. The deep border of the tumor is pointed for clinical cases 1 and 4 whereas a sulcus curvature point is shown for patient 2. In each case, the updated MR images clearly fit the actual US data which 1025 proves the quality of our method.

\section{Discussion}

After comparing our compensation results with existing works, limits and perspectives of our method are discussed. 

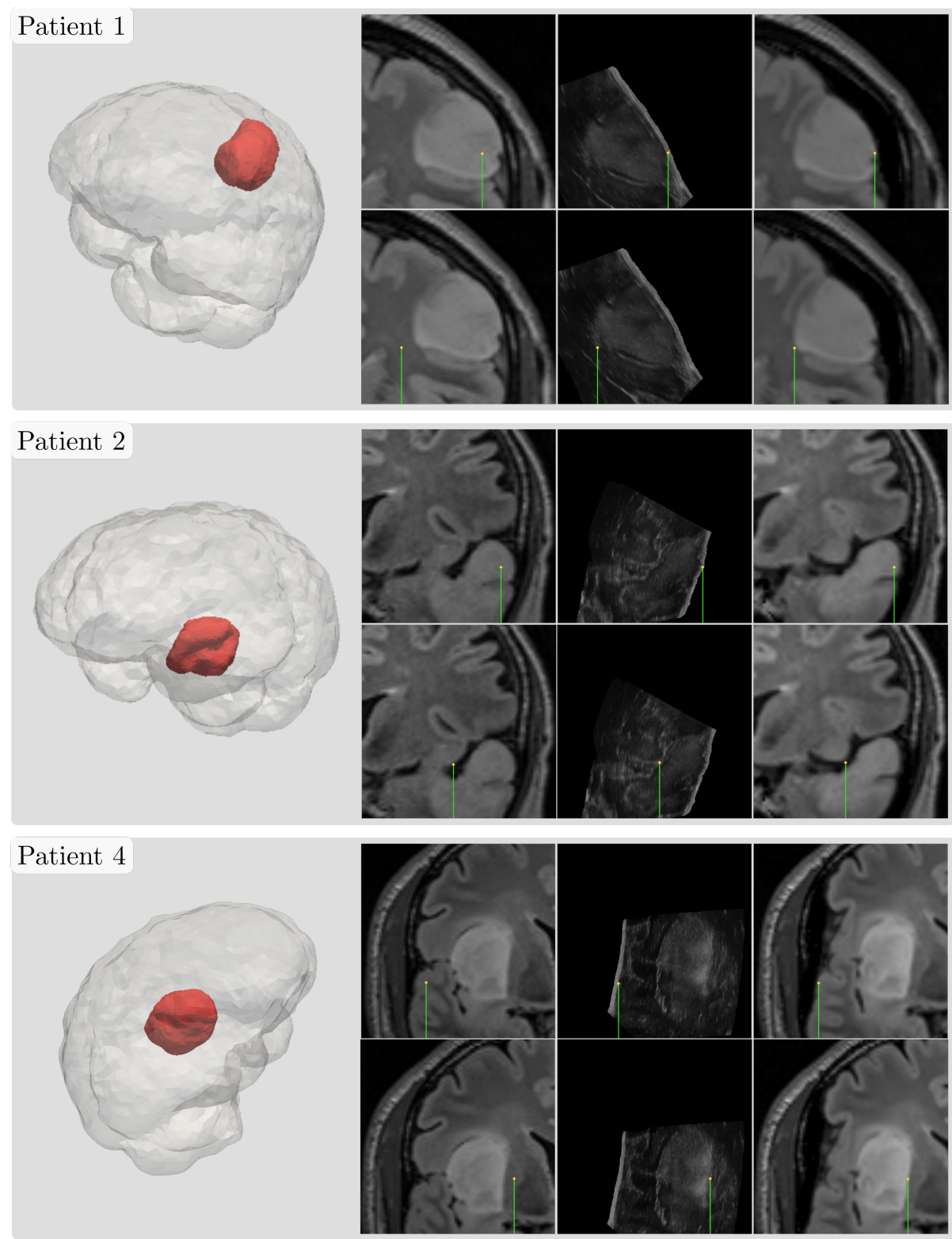

Figure 15: Brain-shift correction for clinical cases 1, 2 and 4. For each patient, a 3D view of its brain and tumor is shown next to medical images. Preoperative MRI (left column), intraoperative b-mode US images (middle column) and updated MRI with our constraint-based compensation method (right column) are displayed within the navigation software CustusX Askeland et al. 2015. A pointer first shows the borders of the exposed cortical surface (top row) then the bottom of the tumor for cases 1 and 4 and a sulcus curvature point for patient 2 (bottom row). 


\subsection{Comparison with existing methods}

topic. However, comparing the accuracy of existing methods remains difficult due to the different input data (US acquisitions, laser range scanner, etc.) and validation protocols proposed in the literature. Consequently, very few authors present a direct comparison between their method and existing ones.

1035 posed by Reinertsen et al. (2014), comparing compensation results on the same surgical cases. While our method cannot be directly compared with other existing techniques, this point can still be discussed. To do so, we have selected recent clinical studies also proposing methods that could be easily embedded into a surgical workflow. Obviously, comparisons have to be carefully interpreted, since results are not presented over the same surgical cases and methods involve different validation protocols.

First, Rivaz \& Collins (2015) introduced an image-based method to register preoperative MRI with intraoperative US images. The registration accuracy is evaluated over 13 patients, based on landmarks set on soft tissues. Their input data (US images) and validation protocol are thus very similar to ours. Results on pre- and post-resection US image registration are presented. However, only the pre-resection ones are accounted for in this paragraph, in order to compare brain-shift compensation results at the same time of the surgery (i.e. just after the opening the dura mater). Initial shifts ranging from 1.5 to $9.4 \mathrm{~mm}$ are reported. These values are in the same order of magnitude as the ones measured on our first four patients, using landmarks set on blood vessels. Rivaz and Collins report results after compensation that range from 1.4 to $4.2 \mathrm{~mm}$, which corresponds to an average correction of $36 \%$ of the shift. For very small initial shifts $(\leq 3.5 \mathrm{~mm})$, computing a percentage of corrected deformation may not be meaningful. Without accounting for these cases, the correction rate increases to $45 \%$. In comparison, this average correction appears globally lower than the $67 \%$ obtained on our cases.

Next, Miga et al. (2015) presented a model-based method using LRS ac- 
quisitions of the cortical surface. Compensation results are evaluated based on shift vectors computed over bitmap images of the craniotomy. Input data and validation process are thus very different from ours, complicating the comparison. In addition, a wider range of measured shift is reported including very large displacements (from 2.5 to $21.3 \mathrm{~mm}$ ). Two reasons explaining such difference could be pointed out. First, the amount of brain-shift is measured after tumor resection, thus accounting for these deformations. Next, it is evaluated on the cortical surface, often showing higher displacements than for deep structures (in our case, the blood vessels). The mean corrected brain-shift is ranging from 0.7 to $4.0 \mathrm{~mm}$, for an average compensation of $69 \%$ of the deformations. Considering only the patients exhibiting an initial shift in the same range of magnitude observed for our cases $(\leq 10 \mathrm{~mm})$, this percentage decreases to $65 \%$. The registration accuracy is thus very similar the one reported in this paper $(67 \%)$.

Finally, Mostayed et al. (2013) and Garlapati et al. (2014) proposed a modelbased method relying on the registration of few points on the exposed cortical surface. In real clinical conditions, these points could be acquired by the surgeon using a tracking pointer tool available in most classic neuronavigation systems. No extra imaging system is thus required which is a strong advantage for the practicality within the operating room. In these papers, the 3D points are however extracted from intraoperative MR images. It is thus very complicated to compare our results to theirs since intraoperative data were not acquired in real surgical conditions. Indeed, the method does not account for the potential calibration errors of the tracking tool nor for the manipulation errors of the surgeon that could substantially affect the compensation results. In addition, compensation results are computed based on objective methods aiming to compare the intraoperative MRI with the generated warped MRI. No global registration scores are thus provided, making a numerical comparison with our results impossible. Nevertheless, similarly to our method these techniques show a registration improvement when using model-based instead of pure image-based approaches. 
As stated above, these comparisons should be cautiously interpreted. While we cannot conclude on an accuracy improvement, they at least show the relevancy and reliability of our registration results, considering the initial and compensated measured brain-shifts. Obviously, the necessity to validate on ex-

tra surgical cases is stressed out, including initial shifts with magnitudes of higher variability. Finally, our method is only evaluated at the opening of the dura mater and its ability to compensate for deformations induced by tumor ablation still has to be proven.

\subsection{Limits and perspectives}

The proposed method has limitations and several aspect could be improved.

Among the parameters gathered in Table 4 , the biomechanical ones are set following the literature. However, high interpersonal differences are highlighted by Sack et al. (2009). Even if these parameters may have a low importance (Wittek et al., 2009), it would be interesting to study their impact on the final registration. Especially, the effect of an heterogeneous elasticity for the tumor has to be evaluated. In addition, these parameters could be estimated intraoperatively using US elastography. While computing true static Young's modulus using shear waves is complex, the ratio between the tumor and surrounding soft tissues elasticity could at least be estimated. Finally, only some of the intrinsic characteristics of the organ are simulated by our brain model (morphology, soft tissues elasticity, contacts with neighbor anatomical structures, etc.). External phenomenons could then be accounted for to improve the method accuracy, such as the gravity-induced stress (Morin et al. 2015).

Like other intraoperative compensation methods, we are sensitive to localization errors (mostly due to probe calibration and image-to-patient registration). Essentially, this was highlighted by our results on the third clinical case. Even if the vessels are well registered, the compensation of the tissue deformations is not satisfying due to the registration errors between the preoperative MRI and intraoperative US images (see Tables 5 and 6). In order to detect and account for these localization errors, a rigid transformation could first be applied on the 
brain model to register the cortical surface with the extracted probe footprint.

Once corrected, the non-rigid tissue deformations could thus be compensated using our constraint-based method

A last limit is the dependency of our method to the presence of vessels around the surgical target. When very few vessels are located close to the tumor for patient 5, our method has difficulties to compensate for brain-shift. Similar results were observed on synthetic data: the method fails to correct the deformation for very sparse input US skeletons (up to $75 \%$ points removed). To overcome this limit, salient corresponding features could be extracted in the soft tissues respectively from the preoperative MR and intraoperative US images. The same constraint-based formalism could be run for the registration of these matching features. Furthermore, our process requires preoperative MRA or another sequence enhanced with contrast agent, to extract the vascular tree. As this may not be a standard protocol for brain tumor surgery, relying on soft tissue features only could enable to spare this additional exam.

\section{Conclusion}

In this paper, a new constraint-based method to compensate for the craniotomy-induced brain-shift observed during tumor ablation procedure was proposed. A solution easily integrable in the operating room, in terms of intraoperative acquisitions (US images), execution times and user interactions is presented. While quantitative improvements over one of the closest methods in the literature (Reinertsen et al. 2014) are shown on five clinical cases, the robustness of the method was also proven using synthetic data. This method addresses some of the current limitations, towards optimal solutions in image-guided neurosurgery. Next stage of this work will be to extend the proposed method in order to compensate for resection-induced brain-shift deformations. 


\section{Acknowledgments}

This work was partly supported by the French ANR within the Investissements d'Avenir program under references ANR-11-LABX-0004 (Labex CAMI)

${ }_{1150}$ and ANR-11-INBS-0006 (Infrastructure d'avenir en Biologie Santé) and by a France-Norway partnership (PHC Aurora 2015/Research Council of Norway). The authors would like to thank Clément Guyomard and Vincent Genty, interns at TIMC-IMAG Laboratory, Grenoble.

\section{Ethic committee}

Anonymous and retrospective images are used for this clinical study. It was approved by the local ethics committee and formal consent was obtained from all individual participants.

\section{References}

Archip, N., Clatz, O., Whalen, S., Kacher, D., Fedorov, A., Kot, A., Chrisochoides, N., Jolesz, F. A., Golby, A. J., Black, P. M., \& Warfield, S. K. (2007). Non-rigid alignment of pre-operative MRI, fMRI, and DT-MRI with intraoperative MRI for enhanced visualization and navigation in image-guided neurosurgery. NeuroImage, 35, 609-624.

Arthofer, C., Morgan, P. S., \& Pitiot, A. (2016). Hierarchical Multi-Atlas Segmentation Using Label-Specific Embeddings, Target-Specific Templates and Patch Refinement. International Workshop on Patch-based Techniques in Medical Imaging, (pp. 84-91).

Askeland, C., Solberg, O. V., Beate, J. B. L., Reinertsen, I., Tangen, G. A., Hofstad, E. F., Iversen, D. H., Vapenstad, C., Selbekk, T., Lango, T., Hernes, T. A., Leira, H. O., Unsgard, G., \& Lindseth, F. (2015). CustusX: an opensource research platform for image-guided therapy. International journal of computer assisted radiology and surgery, 11, 505-519. 
Ayachit, U. (2015). The ParaView Guide: A Parallel Visualization Application.

Kitware.

Benkarim, O. M., Piella, G., Gonzalez Ballester, M. A., \& Sanroma, G. (2016). Enhanced Probabilistic Label Fusion by Estimating Label Confidences Through Discriminative Learning. Medical Image Computing and ComputerAssisted Intervention - MICCAI 2016, 2, 505-512.

Besl, P. J., \& McKay, N. D. (1992). A Method for Registration of 3-D Shapes. IEEE Transactions on Pattern Analysis and Machine Intelligence, 14, 239256.

Bilger, A., Dequidt, J., Duriez, C., \& Cotin, S. (2011). Biomechanical simulation of electrode migration for deep brain stimulation. Medical Image Computing and Computer-Assisted Intervention - MICCAI 2011, 14, 339-346.

Bucki, M., Palombi, O., Bailet, M., \& Payan, Y. (2012). Doppler Ultrasound Driven Biomechanical Model of the Brain for Intraoperative Brain-Shift Compensation: A Proof of Concept in Clinical Conditions. In Soft Tissue Biomechanical Modeling for Computer Assisted Surgery (pp. 135-165). Springer.

Castellano-Smith, A. D., Crum, W. R., Hill, D. L. G., Thacker, N. A., \& Bromiley, P. A. (2003). Biomechanical simulation of atrophy in MR images. Medical Imaging, (pp. 481-490).

CGAL (2016). CGAL User and Reference Manual. (4th ed.). CGAL Editorial Board.

Chatelin, S., Vappou, J., Roth, S., Raul, J. S., \& Willinger, R. (2012). Towards child versus adult brain mechanical properties. Journal of the mechanical behavior of biomedical materials, 6, 166-173.

Chen, I., Coffey, A. M., Ding, S., Dumpuri, P., Dawant, B. M., Thompson, R. C., \& Miga, M. I. (2011). Intraoperative Brain Shift Compensation: Accounting for Dural Septa. IEEE Transcations on Biomedical Engineering, 58, 499 1200 508. 
Clatz, O., Delingette, H., Bardinet, E., Dormont, D., \& Ayache, N. (2003).

Patient-specific biomechanical model of the brain: application to Parkinson's disease procedure. Surgery Simulation and Soft Tissue Modeling, (pp. 321$331)$.

Clatz, O., Delingette, H., Talos, I.-F., Golby, A. J., Kikinis, R., Jolesz, F. A., Ayache, N., \& Warfield, S. K. (2005). Robust Nonrigid Registration to Capture Brain Shift From Intraoperative MRI. IEEE Transactions on Medical Imaging, 24, 1417-1427.

Clements, L. W., Collins, J. A., Weis, J. A., Simpson, A. L., Adams, L. B., ${ }_{1210}$ Jarnagin, W. R., \& Miga, M. I. (2016). Evaluation of model-based deformation correction in image- guided liver surgery via tracked intraoperative ultrasound. Journal of Medical Imaging, 3, 015003.

Courtecuisse, H., Allard, J., Duriez, C., \& Cotin, S. (2011). PreconditionerBased Contact Response and Application to Cataract Surgery. International Conference on Medical Image Computing and Computer-Assisted Intervention, (pp. 315-322).

De Lorenzo, C., Papademetris, X., Staib, L. H., Vives, K. P., Spencer, D. D., \& Duncan, J. S. (2012). Volumetric Intraoperative Brain Deformation Compensation: Model Development and Phantom Validation. IEEE Transactions on Medical Imaging, 31, 1607-1619.

Dequidt, J., Coevoet, E., Thines, L., \& Duriez, C. (2015). Vascular neurosurgery simulation with bimanual haptic feedback. In 12th Workshop on Virtual Reality Interaction and Physical Simulation.

Dumpuri, P., Thompson, R. C., Sinha, T. K., \& Miga, M. I. (2006). Automated 1225 Brain Shift Correction Using A Pre-computed Deformation Atlas. Medical Imaging, (pp. 61411F-61411F-8).

Duriez, C., Dubois, F., Kheddar, A., \& Andriot, C. (2006). Realistic Haptic 
Rendering of Interacting Deformable Objects in Virtual Environments. IEEE transactions on visualization and computer graphics, 12, 36-47.

Faure, F., Duriez, C., Delingette, H., Allard, J., Gilles, B., Marchesseau, S., Talbot, H., Courtecuisse, H., Bousquet, G., Peterlik, I., \& Cotin, S. (2012). SOFA: A Multi-Model Framework for Interactive Physical Simulation. In Soft Tissue Biomechanical Modeling for Computer Assisted Surgery (pp. 283-321). Springer Berlin Heidelberg.

1235 Fedorov, A., Billet, E., Prastawa, M., Gerig, G., Radmanesh, A., Warfield, S. K., Kikinis, R., \& Chrisochoides, N. (2008). Evaluation of Brain MRI Alignment with the Robust Hausdorff Distance Measures. Las Vegas, NV, USA.

Felippa, C. A., \& Haugen, B. (2005). Unified Formulation of Small-Strain Corotational Finite Elements: I. Theory. Computer Methods in Applied Mechanics and Engineering, 194, 2285-2335.

Ferrant, M., Nabavi, A., Macq, B., Black, P. M., Jolesz, F. A., Kikinis, R., \& Warfield, S. K. (2002). Serial registration of intraoperative MR images of the brain. Medical Image Analysis, 6, 337-359.

Ferrant, M., Nabavi, A., Macq, B., Jolesz, F. A., Kikinis, R., \& Warfield, S. K. (2001). Registration of 3-D Intraoperative MR Images of the Brain Using a Finite-Element Biomechanical Model. IEEE Transactions on Medical Imaging, 20, 1384-1397.

Fuerst, B., Wein, W., Müller, M., \& Navab, N. (2014). Automatic ultrasound-MRI registration for neurosurgery using the 2d and 3d LC2 Metric. Medical Image Analysis, (pp. 1312-1319).

Garlapati, R. R., Mostayed, A., Joldes, G. R., Wittek, A., Doyle, B., \& Miller, K. (2015). Towards measuring neuroimage misalignment. Computers in Biology and Medicine, 64, 12-23.

Garlapati, R. R., Roy, A., Joldes, G. R., Wittek, A., Mostayed, A., Doyle, B., 1255 Warfield, S. K., Kikinis, R., Knuckey, N., Bunt, S., \& Miller, K. (2014). More 
accurate neuronavigation data provided by biomechanical modeling instead of rigid registration. Journal of neurosurgery, 120, 1477-1483.

Gerard, I. J., Kersten-Oertel, M., Petrecca, K., Sirhan, D., Hall, J. A., \& Collins, D. L. (2017). Brain shift in neuronavigation of brain tumors: A review. Medical Image Analysis, 35, 403-420.

Hastreiter, P., Rezk-Salama, C., Soza, G., Bauer, M., Greiner, G., Fahlbush, R., Ganslandt, O., \& Nimsky, C. (2004). Strategies for brain-shift evaluation. Medical Image Analysis, 8, 447-464.

Hill, D. L. G., Maurer Jr, C. R., Maciunas, R. J., Barwise, J. A., Fitzpatrick, M. J., \& Wang, M. Y. (1998). Measurement of intraoperative brain surface deformation under a craniotomy. Neurosurgery, 43, 514-526.

Hu, J., Jin, X., Lee, J. B., Zhang, L., Chaudary, V., Guthikonda, M., Yang, K. H., \& King, A. I. (2007). Intraoperative brain shift prediction using a 3d inhomogeneous patient-specific finite element model. Journal of neurosurgery, $106,164-169$.

Ji, S., Wu, Z., Hartov, A., Roberts, D. W., \& Paulsen, K. D. (2008). Mutualinformation-based image to patient re-registration using intraoperative ultrasound in image-guided neurosurgery. Medical physics, 35.

Joldes, G. R., Wittek, A., \& Miller, K. (2009). Suite of finite element algorithms for accurate computation of soft tissue deformation for surgical simulation. Medical Image Analysis, 13, 912-919.

Joldes, G. R., Wittek, A., \& Miller, K. (2010). Real-time nonlinear finite element computations on GPU - Application to neurosurgical simulation. Computer Methods in Applied Mechanics and Engineering, 199, 3305-3314.

Kaster, T., Sack, I., \& Samani, A. (2011). Measurement of the hyperelastic properties of ex vivo brain tissue slices. Journal of Biomechanics, 44, 11581163. 
Kruse, S. A., Dresner, M. A., Rossman, P. J., Felmlee, J. P., Jack, C. R., \& Ehman, R. L. (1999). Palpation of the Brain Using Magnetic Resonance Elastography. International Society for Magnetic Resonance in Medicine, (p. 258).

Kruse, S. A., Rose, G. H., Glaser, K. J., Manduca, A., Felmlee, J. P., Jack Jr., C. R., \& Ehman, R. L. (2008). Magnetic resonance elastography of the brain. NeuroImage, 39, 231-237.

Li, M., Wittek, A., \& Miller, K. (2014). Efficient Inverse Isoparametric Mapping Algorithm for Whole-Body Computed Tomography Registration Using Deformations Predicted by Nonlinear Finite Element Modeling. Journal of Biomechanical Engineering, 136, 084503.

McCracken, P. J., Manduca, A., Felmlee, J. P., \& Ehman, R. L. (2005). Mechanical Transient-Based Magnetic Resonance Elastography. Magnetic Resonance in Medicine, 53, 628-639.

Mercier, L., Fonov, V., Haegelen, C., Del Maestro, R. F., Petrecca, K., \& Collins, D. L. (2012). Comparing two approaches to rigid registration of threedimensional ultrasound and magnetic resonance images for neurosurgery. International journal of computer assisted radiology and surgery, 7, 125-136.

Miga, M. I., Sun, K., Chen, I., Clements, L. W., Pheiffer, T. S., Simpson, A. L., \& Thompson, R. C. (2015). Clinical evaluation of a model-updated image-guidance approach to brain shift compensation: experience in 16 cases. International journal of computer assisted radiology and surgery, 11, 14671474 .

Miller, K., \& Chinzei, K. (2002). Mechanical properties of brain tissue in tension. Journal of Biomechanics, 35, 483-490.

Miller, K., Horton, A., Joldes, G. R., \& Wittek, A. (2012). Beyond Finite Elements: A Comprehensive, Patient-Specific Neurosurgical Simulation Utilizing a Meshless Method. Journal of Biomechanics, 45, 2698-2701. 
Miller, K., Joldes, G. R., Lance, D., \& Wittek, A. (2007). Total Lagrangian explicit dynamics finite element algorithm for computing soft tissue deformation. COMMUNICATIONS IN NUMERICAL METHODS IN ENGINEERING, 23, 121-134.

Miller, K., \& Lu, J. (2013). On the prospect of patient-specific biomechanics without patient-specific properties of tissues. Journal of the mechanical behavior of biomedical materials, 27, 154-166.

Mohammadi, A., Ahmadian, A., Darbandi Azar, A., Darban Sheykh, A., Amiri, F., \& Alirezaie, J. (2015). Estimation of intraoperative brain shift by combination of stereovision and doppler ultrasound: phantom and animal model study. International journal of computer assisted radiology and surgery, 10, 1753-1764.

Morin, F., Chabanas, M., Courtecuisse, H., \& Payan, Y. (2017). Biomechanical modeling of brain soft tissues for medical applications. In Biomechanics of Living Organs: Hyperelastic Laws for Finite Element Modeling.

Morin, F., Courtecuisse, H., Chabanas, M., \& Payan, Y. (2015). Rest shape computation for highly deformable model of brain. Computer Methods in Biomechanics and Biomedical Engineering, 18, 2006-2007.

Mostayed, A., Garlapati, R. R., Joldes, G. R., Wittek, A., Roy, A., Kikinis, ${ }_{1330}$ R., Warfield, S. K., \& Miller, K. (2013). Biomechanical Model as a Registration Tool for Image-Guided Neurosurgery: Evaluation Against BSpline Registration. Annals of Biomechanical Engineering, 41, 2409-2425.

Müller, M., Dorsey, J., McMillan, L., Jagnow, R., \& Cutler, B. (2002). Stable real-time deformations. Proceedings of ACM SIGGRAPH Symposium on ${ }_{1335}$ Computer Animation (SCA), (pp. 49-54).

Nabavi, A., Black, P. M., Gering, D. T., Westin, C.-F., Mehta, V., Pergolizzi, R. S., Ferrant, M., Warfield, S. K., Hata, N., Schartz, R. B., Wells, W. M., 
Kikinis, R., \& Jolesz, F. A. (2001). Serial Intraoperative Magnetic Resonance Imaging of Brain Shift. Neurosurgery, 48, 787-798.

1340 Nakajima, S., Atsumi, H., Kikinis, R., Moriarty, T. M., Metcalf, D. C., Jolesz, F. A., \& Black, P. M. (1997). Use of cortical surface vessel registration for image-guided neurosurgery Neurosurgery. Neurosurgery, 40, 1201-1210.

Nesme, M., Payan, Y., \& Faure, F. (2005). Efficient, physically plausible finite elements. Eurographics, .

1345 Nimsky, C., Ganslandt, O., Cerny, S., Hastreiter, P., Greiner, G., \& Fahlbush, R. (2000). Quantification of, Visualization of, and Compensation for Brain Shift Using Intraoperative Magnetic Resonance Imaging. Neurosurgery, 47, 1070-1080.

Nimsky, C., Ganslandt, O., Hastreiter, P., \& Fahlbush, R. (2001). Intraoperative 1350 Compensation for Brain Shift. Surgical Neurology, 56, 357-364.

Paulsen, K. D., Miga, M. I., Kennedy, F. E., Hoopes, J., Hartov, A., \& Roberts, D. W. (1999). A Computational Model for Tracking Subsurface Tissue Deformation During Stereotactic Neurosurgery. IEEE Transactions on Biomedical Engineering, 46, 213-225.

1355 Pereira, V. M., Smith-Ockeloen, I., Brina, O., Babic, D., Breeuwer, M., Schaller, K., Lovblad, K.-O., \& Ruijters, D. (2016). Volumetric Measurements of Brain Shift Using Intraoperative Cone-Beam Computed Tomography: Preliminary Study. Operative Neurosurgery, 12, 4-13.

Prastawa, M., Bullit, E., \& Gerig, G. (2009). Simulation of brain tumors in MR images for evaluation of segmentation efficacy. Medical Image Analysis, 13, $297-311$.

Reinertsen, I., Descoteaux, M., Siddiqi, K., \& Collins, D. L. (2007a). Validation of vessel-based registration for correction of brain-shift. Medical Image Analysis, 11, 374-388. 
Reinertsen, I., Lindseth, F., Askeland, C., Iversen, D. H., \& Unsgard, G. (2014). Intra-operative correction of brain-shift. Acta Neurochirurgica, 156, 13011310.

Reinertsen, I., Lindseth, F., Unsgard, G., \& Collins, D. L. (2007b). Clinical validation of vessel-based registration for correction of brain-shift. Medical Image Analysis, 11, 673-684.

Rivaz, H., \& Collins, D. L. (2015). Deformable registration of preoperative $\mathrm{MR}$, pre-resection ultrasound, and post-resection ultrasound images of neurosurgery. International journal of computer assisted radiology and surgery, 10, 1017-1028.

1375 Roberts, D. W., Hartov, A., Kennedy, F. E., Miga, M. I., \& Paulsen, K. D. (1998). Intraoperative Brain Shift and Deformation: A Quantitative Analysis of Cortical Displacement in 28 Cases. Neurosurgery, 43, 749-758.

Sack, I., Beierbach, B., Wuerfel, J., Dieter, K., Hamhaber, U., Papazoglou, S., Martus, P., \& Braun, J. (2009). The impact of aging and gender on brain viscoelasticity. NeuroImage, 46, 652-657.

Sase, K., Fukuhara, A., Tsujita, T., \& Konno, A. (2015). GPU-accelerated surgery simulation for opening a brain fissure. Robomech Journal, 2, 1-16.

Schiavone, P., Chassat, F., Boudou, T., Promayon, E., Valdivia, F., \& Payan, Y. (2009). In vivo measurement of human brain elasticity using a light aspiration device. Medical Image Analysis, 13, 673-678.

Schroeder, W., Martin, K., \& Lorensen, B. (2006). The Visualization Toolkit (4th ed.). Kitware.

Shakeri, M., Ferrante, E., Tsogkas, S., Lippe, S., Kadoury, S., Kokkinos, I., \& Paragios, N. (2016). Prior-based Coregistration and Cosegmentation. Medical Image Computing and Computer-Assisted Intervention - MICCAI 2016, 2, $529-537$. 
Smith, S. M. (2002). Fast Robust Automated Brain Extraction. Human Brain Mapping, 17, 143-155.

Sun, H., Lunn, K. E., Farid, H., Wu, Z., Roberts, D. W., Hartov, A., \& Paulsen, K. D. (2005a). Stereopsis-Guided Brain Shift Compensation. IEEE Transactions on Medical Imaging, 24, 1039-1052.

Sun, H., Roberts, D. W., Farid, H., Wu, Z., Hartov, A., \& Paulsen, K. D. (2005b). Cortical Surface Tracking Using a Stereoscopic Operating Microscope. Neurosurgery, 56, 86-97.

Sun, K., Pheiffer, T. S., Simpson, A. L., Weis, J. A., Thompson, R. C., \& Miga, M. I. (2014). Near Real-Time Computer Assisted Surgery for Brain Shift Correction Using Biomechanical Models. IEEE Journal of Translational Engineering in Health and Medicine, 2, 1-13.

Taha, A. A., \& Hanbury, A. (2015). Metrics for evaluating 3d medical image segmentation: analysis, selection, and tool. BMC Medical Imaging, 15, 29.

Tavner, A., Roy, T. D., Hor, K., Majimbi, M., Joldes, G. R., Wittek, A., Bunt, S., \& Miller, K. (2016). On the appropriateness of modelling brain parenchyma as a biphasic continuum. Journal of the mechanical behavior of biomedical materials, 61, 511-518.

Uffmann, K., Maderwald, S., de Greiff, A., \& Ladd, M. E. (2004). Determination of Gray and White Matter Elasticity with MR Elastography. Proc. Intl. Soc. Mag. Reson. Med, 11 .

Valencia, A., Benjamin, B., \& Ortega, J. H. (2012). Modeling of Brain Shift Phenomenon for Different Craniotomies and Solid Models. Journal of Applied Mathematics, 2012.

Vermandel, M., Betrouni, N., Taschner, C., Vasseur, C., \& Rousseau, J. (2007). From MIP to MRA segmentation using fuzzy set theory. Computerized Medical Imaging and Graphics, 31, 128-140. 
Vigneron, L. M., Noels, L., Warfield, S. K., Verly, J. G., \& Robe, P. A. (2012). Serial FEM/XFEM-Based Update of Preoperative Brain Images Using Intraoperative MRI. International Journal of Biomedical Imaging, 2012, 872783.

Wan, M., Liang, Z., Ke, Q., Hong, L., Bitter, I., \& Kaufman, A. (2002). Automatic Centerline Extraction for Virtual Colonoscopy. IEEE Transactions on Medical Imaging, 21, 1450-1460.

Wittek, A., Hawkins, T., \& Miller, K. (2009). On the unimportance of constitutive models in computing brain deformation for image-guided surgery. Biomechanics and modeling in mechanobiology, 8, 77-84.

Wittek, A., Miller, K., Kikinis, R., \& Warfield, S. K. (2007). Patient-specific model of brain deformation: Application to medical image registration. Journal of Biomechanics, 40, 919-929.

Yousefi, H., Ahmadian, A., Khodadad, D., Saberi, H., \& Daneshmehr, A. (2013). An optimised linear mechanical model for estimating brain shift caused by meningioma tumours. International Journal of Biomedical Science and Engineering, 1, 1-9.

1435 Yushkevich, P. A., Piven, J., Cody Hazlett, H., Gimpel Smith, R., Ho, S., Gee, J. C., \& Gerig, G. (2006). User-guided 3d active contour segmentation of anatomical structures: Significantly improved efficiency and reliability. NeuroImage, 31, 1116-1128.

Zhang, J. Y., Joldes, G. R., Wittek, A., \& Miller, K. (2013). Patient-specific computational biomechanics of the brain without segmentation and meshing. International journal for numerical methods in biomedical engineering, 29, 293-308. 